\title{
Acoustic scattering by benthic and planktonic shelled animals
}

Timothy K. Stanton and Dezhang Chu

Department of Applied Ocean Physics and Engineering, Woods Hole Oceanographic Institution, Woods Hole, Massachusetts 02543

Peter H. Wiebe

Department of Biology, Woods Hole Oceanographic Institution, Woods Hole, Massachusetts 02543

Robert L. Eastwood and Joseph D. Warren

Department of Applied Ocean Physics and Engineering, Woods Hole Oceanographic Institution, Woods Hole, Massachusetts 02543

(Received 14 September 1998; revised 27 September 1999; accepted 27 October 1999)

Acoustic backscattering measurements and associated scattering modeling were recently conducted on a type of benthic shelled animal that has a spiral form of shell (Littorina littorea). Benthic and planktonic shelled animals with this shape occur on the seafloor and in the water column, respectively, and can be a significant source of acoustic scattering in the ocean. Modeling of the scattering properties allows reverberation predictions to be made for sonar performance predictions as well as for detection and classification of animals for biological and ecological applications. The studies involved measurements over the frequency range $24 \mathrm{kHz}$ to $1 \mathrm{MHz}$ and all angles of orientation in as small as $1^{\circ}$ increments. This substantial data set is quite revealing of the physics of the acoustic scattering by these complex shelled bodies and served as a basis for the modeling. Specifically, the resonance structure of the scattering was strongly dependent upon angle of orientation and could be traced to various types of rays (e.g., subsonic Lamb waves and rays entering the opercular opening). The data are analyzed in both the frequency and time domain (compressed pulse processing) so that dominant scattering mechanisms could be identified. Given the complexity of the animal body (irregular elastic shell with discontinuities), approximate scattering models are used with only the dominant scattering properties retained. Two models are applied to the data, both approximating the body as a deformed sphere: (1) an averaged form of the exact modal-series-based solution for the spherical shell, which is used to estimate the backscattering by a deformed shell averaged over all angles of orientation, and produces reasonably accurate predictions over all $k_{1} a_{\mathrm{esr}}\left(k_{1}\right.$ is the acoustic wave number of the surrounding water and $a_{\text {esr }}$ is the equivalent spherical radius of the body), and (2) a ray-based formula which is used to estimate the scattering at fixed angle of orientation, but only for high $k_{1} a_{\text {esr }}$. The ray-based model is an extension of a model recently developed for the shelled zooplankton Limacina retroversa that has a shape similar to that of the Littorina littorea but swims through the water [Stanton et al., J. Acoust. Soc. Am. 103, 236-253 (1998b)]. Applications of remote detection and classification of the seafloor and water column in the presence of shelled animals are discussed. (C) 2000 Acoustical Society of America. [S0001-4966(00)01702-1]

PACS numbers: 43.30.Ft, 43.30.Sf, 43.30.Hw, 43.20.Fn [DLB]

\section{LIST OF SYMBOLS}

1,2

subscripts indicating medium " 1 " (surrounding fluid) and medium " 2 "' (body medium)

radius of sphere

average radius of an irregular sphere

equivalent spherical radius-radius of sphere

that has same volume as object of interest

related to dispersion of Lamb wave

modal-series coefficient for spherical shell

$b_{m}$

$\beta_{L}$

$c_{1}, c_{2}$

$c_{L}$

$\bar{c}_{L}$ attenuation coefficient of Lamb wave on elastic shelled sphere sound speed for medium " 1 "' (surrounding fluid) and medium ' 2 "' (body medium) sound speed of Lamb wave $c_{L}$ evaluated for $k_{1} \bar{a}$
$\Delta_{a}$ $f$

$f_{b s}$

$f_{\text {spec }}$,

$f_{\text {op }}, f_{\text {Lamb }}$

$\mathcal{F}$

$F_{\text {spec }}$,

$F_{\text {op }}, F_{L}$

$g$

$G_{L}$ deviation in effective radius from mean radius of rough sphere

scattering amplitude

scattering amplitude in backscattering direction components of $f_{b s}$ from front interface

("specular wave"), opercular opening of shell, and in association with Lamb wave, respectively packing factor for scatterers on the seafloor $[=($ area covered by scatterers $) /($ total area of seafloor)] empirical factors, ranging in value from 0 to 1 , to account for loss of each respective wave (specular, opercular, Lamb) due to discontinuity in shell

$\rho_{2} / \rho_{1}$ coupling coefficient for combination of landing 
and launching of Lamb waves on shell $h$

$h c \quad h$ for compressional sound speed in elastic shell

$h s \quad h$ for shear sound speed in elastic shell

$i \quad \sqrt{-1}$

$k_{1} \quad$ acoustic wave number $\left(=2 \pi / \lambda_{1}\right)$ in medium " 1 " (surrounding fluid)

$\lambda_{1}$ acoustic wavelength in medium " 1 "' (surrounding fluid)

$P_{\text {inc }} \quad$ incident pressure at the object

$\Phi_{L} \quad$ phase shift of Lamb wave heuristically added for nonideal body

$\rho_{1}, \rho_{2} \quad$ mass density in medium " 1 "' (surrounding fluid) and medium ' 2 '" (body medium)

\section{$p_{\text {scat }} \quad$ scattered pressure}

$\mathcal{R}_{12}$

RTS

$S_{A}$

$\sigma_{r}$

$\sigma_{b s}$

$T S$

$\theta_{g}$

$\theta_{L}$

$\langle\ldots\rangle$ plane wave/plane interface reflection coefficient [reflection off of medium " 2 " (body medium) due to incident beam in medium " 1 " (surrounding fluid $)]\left[=\left(\rho_{2} c_{2} / \rho_{1} c_{1}-1\right) /\left(\rho_{2} c_{2} / \rho_{1} c_{1}\right.\right.$ $+1)]$

reduced target strength

area scattering strength differential backscattering cross section target strength

grazing angle $\left(\theta_{g}=90^{\circ}\right.$ corresponds to normal incidence)

launch/land angle for Lamb wave

average over ensemble of statistically independent samples
$\left\langle\Delta_{a}^{2}\right\rangle^{1 / 2}$ (roughness parameter of irregular shell)

\section{INTRODUCTION}

Marine organisms can sometimes be a significant source of acoustic scattering. The scattering properties of the animals need to be understood for two major reasons: (1) Sonar performance prediction. When designing a sonar system, all sources of reverberation, i.e., unwanted scattered signals, need to be modeled so that the performance of the system can be predicted in terms of the signal-to-reverberation ratio. The reverberation from marine life can sometimes cause false alarms in active sonar detection systems. (2) Bioacoustical oceanography. Biologists can take advantage of the fact that certain marine life scatters sound and use acoustic systems to survey the animals. The scattering needs to be modeled so that the echo data from the survey can be interpreted in terms of meaningful biological parameters such as length and numerical density.

There is a wide range of animals that live in the ocean, including fish and zooplankton that live in the water column and the animals that live in the benthic (seafloor) zone. The fish and planktonic animals will contribute to acoustic scattering from the water column while the benthic animals will contribute to scattering by the seafloor. There are thousands of species present in the ocean and it would not be practical to model the scattering by each animal on a species-byspecies basis. It has been convenient to characterize the animals according to their gross anatomical group. For example, in a recent study, zooplankton were grouped according to whether they were weak scatterers, elastic shelled bodies, or gas-bearing (Stanton et al., 1994, 1996, 1998a, 1998b). Fish are sometimes characterized according to whether or not they have a swimbladder (Foote, 1980).

Shelled animals are present both in the water column and on the seafloor. For example, pteropods are a group of gastropod molluscar zooplankton that swim throughout the water column and other gastropod groups reside on the seafloor. There has been significant evidence that these animals can influence or even dominate the volume and seafloor reverberation, respectively, when present (Wiebe et al., 1996, 1997; Jackson et al., 1986; Stanic et al., 1989; Zhang, 1996). The main issue in modeling the acoustic scattering by the shelled bodies is that their shape is so complex. In addition,

few measurements of the material properties are available for use in the modeling. There are no exact (analytical) mathematical solutions available to describe the scattering, so approximate analytical or numerical approaches must be used. Further complicating the issue is the fact that, even if a general solution were known, the shape of the shells is sufficiently complex that the characterization of the morphology is a challenge.

In a recent study, the acoustic scattering properties of several major zooplankton groups were studied and models developed (Stanton et al., 1998b). One of these groups involved the planktonic shelled gastropod (Limacina retro$v e r s a$ ). This is a thin-shelled snail that swims throughout the water column, can occur at numerical densities of up to thousands per cubic meter, and can (collectively) produce significant reverberation levels (Wiebe et al., 1996, 1997). At-sea measurements of the scattering by the animals were made in a laboratory-style tank shortly after they were caught. Broadband signals were used to investigate the resonance scattering structure and scattering models were developed. Key scattering mechanisms were identified in this high $k_{1} a_{\text {esr }}$ region: there was significant evidence that a subsonic Lamb wave was the source of some of the structure observed (Stanton et al., 1998b) ( $k_{1}$ is the wave number of the surrounding water and $a_{\mathrm{esr}}$ is the equivalent spherical radius of the irregular body, which is the radius of a sphere with the same volume as the irregular body). Furthermore, the structure and overall scattering levels changed with orientation, which indicated that the irregularities played a major role in the scattering. Significant progress was made toward the understanding of scattering by complex shelled bodies in this study. However, due to the time constraints of performing the research at sea, the experiments were naturally limited by the fact that the full range of orientations and acoustic frequencies could not be realized for all animals (it was essential to perform these experiments at sea as it allowed studies to be performed on freshly caught animals spanning a wide range of species not available near the coast). Furthermore, only a limited class of species was used. The next logical step in this research is to extend this type of study to a wider range of frequencies and orientations so that the physics of the scattering process can be understood over a wider range of 
conditions. Also, it is important to examine other classes of animals within a gross anatomical group.

We have just completed an extensive set of acoustic backscattering measurements involving benthic shelled bodies. The experiments involved a wide range of acoustic frequencies spanning from $k_{1} a_{\text {esr }}$ much less than unity to $k_{1} a_{\text {esr }}$ much greater than unity and the full range of orientation angles in one plane. The animals were chosen to fit two criteria: (1) to be from the seafloor and (2) to have a shell with a morphology similar to that of the planktonic ones studied so that direct comparisons (or simple extrapolations) could be made. With this high-quality data set, dominant scattering mechanisms can be clearly identified with the scattering geometry. The high $k_{1} a_{\text {esr }}$ scattering model initially developed for the planktonic ones described above is extended and applied to these benthic animals. Furthermore, an all $-k_{1} a_{\text {esr }}$ model is applied to the benthic animals for data averaged over all angles of incidence. Similarities between the scattering by the two animal types have been determined and connections between the scattering model predictions and applications to volume and seafloor scattering when shelled bodies are present are summarized.

This paper is organized as follows. In Sec. I, basic definitions and scattering models are presented involving both the ray-based and modal-series-based models. Section II describes the experiment and Secs. III and IV present the results of the experiment and comparisons between the models and some of the results. Applications of the laboratory-based results to surveys in the ocean of the water column and seafloor are discussed in Sec. V.

\section{THEORY}

The targets under investigation are irregular shelled bodies with discontinuities. There are no exact analytical solutions that can describe the scattering by these bodies, therefore approximate or numerical solutions must be explored. After a section defining basic scattering terms, two approximate approaches are summarized. One involves an average of the exact modal-series solution to the spherical shell which has application to the problem for all $k_{1} a_{\mathrm{esr}}$, and the other involves a ray-based approach which is valid for only the high $k_{1} a_{\text {esr }}$ region (i.e., $k_{1} a_{\text {esr }}$ greater than unity).

\section{A. Definitions}

The far-field backscattered energy from a bounded body is commonly expressed in terms of the target strength TS, which is a logarithmic form of the scattering amplitude $f$ evaluated in the backscattering direction. The scattering amplitude describes the efficiency with which an object scatters sound and is defined in terms of the incident and scattered pressures $P_{\text {inc }}$ and $p_{\text {scat }}$ as

$$
p_{\text {scat }}=P_{\text {inc }} \frac{e^{i k_{1} r}}{r} f,
$$

where $r$ is the distance between the object and receiver. This expression is associated with a single echo or realization from a given individual target. With the geometry and source/receiver terms listed separately in the above equation, the scattering amplitude contains all of the scattering physics related to the target itself. The amplitude depends upon the object size, shape, orientation, and material properties as well as the acoustic wavelength. Scattering modeling efforts result in derivations or descriptions of $f$.

The target strength is defined in terms of the backscattering amplitude $f_{b s}$ as

$$
\mathrm{TS}=10 \log \left|f_{b s}\right|^{2}=10 \log \sigma_{b s},
$$

where $f_{b s}$ is $f$ evaluated in the backscattering direction and the differential backscattering cross section $\sigma_{b s}$ is defined as $\sigma_{b s}=\left|f_{b s}\right|^{2}$ (this differential cross section should not be confused with the commonly used backscattering cross section $\sigma$ where $\left.\sigma=4 \pi \sigma_{b s}\right)$. The target strength is in units of decibels relative to $1 \mathrm{~m}^{2}$.

In order to compare the scattering by objects of the same shape but of different sizes, the target strength is normalized according to the square of some dimension. The normalized or reduced target strength (RTS) for the case of the ideal sphere is defined in terms of the TS and radius $a$ of the sphere as

$$
\mathrm{RTS}=\mathrm{TS}-10 \log \pi a^{2} .
$$

For the case of irregular shelled animals, the equivalent spherical radius, $a_{\text {esr }}$, can be used in Eq. (3).

For the case involving multiple targets or multiple realizations of the same target, it is useful to study the average echo energy. At high enough frequencies or short enough wavelengths, the phases of the echoes from the targets will be random with respect to each other and the average echo energy from the aggregation will be equal to the sum of the average echo energy from each individual (averaged over independent realizations). The equality holds true only when effects due to multiple scattering such as extinction and higher-order scattering (beyond first order) are negligible. Since the backscattering cross section is proportional to echo energy, the "average" target strength of a target is commonly expressed in terms of the average value of the backscattering cross section

$$
\langle\mathrm{TS}\rangle=10 \log \left\langle\sigma_{b s}\right\rangle,
$$

where the averaging process, denoted by the brackets $\langle\ldots$.$\rangle ,$ was performed before the logarithm operation was taken. The averaging is over an ensemble of statistically independent realizations of one or more variables such as animal size and/or orientation.

\section{B. Modal-series solution to spherical shell-all $k_{1} a_{\mathrm{esr}}$}

Although there are no exact analytical solutions to the scattering by these irregular shelled bodies, there are exact solutions to objects with certain simple shapes. It is useful to explore exact solutions involving simple shapes in order to determine insight into the scattering physics as well as conditions under which they provide a reasonable approximation to the more complex shapes. There exists an exact solution to the spherical shell. Although the animals are somewhat elongated (ratio of length to width is approximately 1.6) and irregular with discontinuities, there is some utility to investigating this solution, especially in light of the fact that there 
is not an exact analytical solution to the shape that more resembles the animals. There will be some common elements in the scattering processes of both the sphere and the animals, especially since most of the animal's shell is closed and continuous (as opposed to the open half shell of a clam). At the high values of $k_{1} a$ (where $a$ is the radius of the outer boundary of the spherical shell), there will be scattering from the front curved interface and surface elastic waves. Both the sphere and the animal will have both types of processes. There will, however, be distinct differences in the structure and overall values involving single realizations because of the variability of the local radius of curvature and shell thickness of the animal as well as the discontinuities. At the lower values of $k_{1} a$, the scattering will be more a function of the volume of the shell and there is more potential for applicability of the sphere solution.

The backscattering amplitude for the spherical shell is given in general form as

$$
f_{b s}=\frac{-i}{k_{1}} \sum_{m=0}^{\infty}(-1)^{m}(2 m+1) b_{m} .
$$

The term $b_{m}$ is the modal-series coefficient and can be expressed in terms of the ratio of two $6 \times 6$ determinants based upon the boundary conditions. The radius of the shell, $a$, is implicit in $b_{m}$. The solution was first derived in Goodman and Stern (1962) and is exact over all $k_{1} a$.

The modal-series solution to the sphere was transformed by others into a ray solution (Überall, 1973; Marston, 1992) in which the scattering amplitude was expressed in terms of an infinite series of rays. The amplitude and phase of each ray were determined through the Sommerfeld-Watson Transformation (SWT). One of those forms of the SWT was used in Stanton et al. (1998b) as a basis of modeling the scattering by planktonic shelled animals (see also discussion in Sec. IC). In that analysis, the ray solution was (coherently) averaged over an ensemble of sizes to emulate the effect of the multiple ray paths around the irregular body for a single realization of animal size. Using that same approach, the modal-series solution could be averaged to obtain a similar effect. The ray approach has great utility since various rays that appear explicitly in the formulation can be given different weighting according to the physical process specific to the irregular body [Stanton et al. (1998b)]. In contrast, these rays do not appear explicitly in the modal-series solution. The ray approach, however, is only valid in the high $k_{1} a$ region. Thus although the modal-series approach may not be as useful in the high $k_{1} a$ region as the ray approach, the solution provides predictions (of varying accuracy) over the whole range of $k_{1} a$.

In the application to the shelled bodies in this paper, the modal-series solution will be averaged over a range of sizes and shell thickness in order to estimate the scattering by the animals averaged over angle of orientation. The average increases the number of ray paths and reduces differences in structure of the predictions and data. This approach was also generally successful in applying an ensemble average of the exact solution for the solid elastic sphere to the scattering by individual sand grains and suspended sediment (Thorne et al., 1993, 1995).

\section{Approximate ray solution to irregular shell- $k_{1} a_{\mathrm{esr}}$ $\gtrsim 1$ (geometric scattering region)}

A convenient method for modeling the scattering by the irregular shelled bodies involves the use of a ray approach. The formulation can be constructed using the dominant scattered rays and can also be manipulated analytically. There is great potential with a ray solution to be able to predict the resonance structure of the scattering. The disadvantage is the fact that ray solutions are generally only valid in the high $k_{1} a_{\text {esr }}$ region, or geometric scattering region.

There is a range of sophisticated generalized ray theories that can be used, including those described in Felsen and $\mathrm{Lu}$ (1989), Ho and Felsen (1990), Norris and Rebinsky (1994), Ho (1994), Yang et al. (1995), Rebinsky and Norris (1995), and Yang et al. (1996). Included in those formulations are arbitrary coupling coefficients between the incident wave and the circumferential waves, and arbitrary ray paths due to the irregular surface of the body.

In a recent paper, we derived a simplified formula based upon a limited set of data involving the planktonic gastropod Limacina retroversa (Stanton et al., 1998b). The formula used a single coupling coefficient associated with an average radius of the body. The random phases of the circumnavigating Lamb waves were estimated through assignment of a randomized radius of curvature of the body. As an approximation, each wave was constructed so as to follow the path of a great circle.

Included in that formulation were two terms associated with rays caused by the scattering by the front interface of the body and by a Lamb wave circumnavigating the body. These terms were chosen as there was evidence that both existed sometimes with substantial energy. Our much more extensive set of data presented herein involving the benthic gastropod snail (Littorina littorea), which is morphologically similar to Limacina, indicate that for certain orientations, there is also a ray that propagates into the opercular opening and scatters back toward the receiver. We therefore extend the formula presented in Stanton et al. (1998b) to

$$
f_{b s} \simeq f_{\text {spec }}+f_{\text {op }}+f_{\text {Lamb }},
$$

where the terms $f_{\text {spec }}$ and $f_{\text {op }}$ represent the scattering from the front interface and back of the opercular opening, respectively, and $f_{\text {Lamb }}$ is the Lamb wave that circumnavigates the body.

This addition of the term $f_{\text {op }}$, in essence takes the place of one of the terms $f_{t w}$ that is discussed in that paper and ignored. That term was originally intended to be a transmitted wave that passed through the shell, reflected off of various interfaces beyond the front interface, and then returned to the receiver. Since the shell is hard, the term was considered to be small. However, for the case in which the opercular opening is facing the sonar system, there is no boundary for the ray to pass through at the opening and the bundle of rays associated with the opening will pass into the shell unperturbed. Also, the specular wave $f_{\text {spec }}$ and wave associated with the opercular opening $f_{\text {op }}$ interfere in a manner consistent with waves reflecting off of two points separated by a distance comparable to the diameter of the body. There are other phenomena that can also give rise to such an effect, but 
have not been included because they are generally of such low energy. They are the waves scattered by the tip and edge of the opercular opening. It is calculated that the tip scatters sound with a target strength of about $-90 \mathrm{~dB}$, which is 10 's of $\mathrm{dB}$ lower than the specular return from the curved shell face (Bowman et al., 1987). The backscattered echo from the edge at these very high frequencies is 10 's of $\mathrm{dB}$ lower than the specular return when the sound is well off normal incidence from the local plane of the edge (Jebsen and Medwin, 1982).

The various terms in the above equation are given as

$$
\begin{aligned}
& f_{\mathrm{spec}} \simeq \frac{a_{\mathrm{spec}}}{2} \mathcal{R}_{12} F_{\mathrm{spec}} e^{-i 2 k_{1} a_{\mathrm{spec}}}, \\
& f_{\mathrm{op}} \simeq \frac{1}{2} a_{\mathrm{op}} \mathcal{R}_{12} F_{\mathrm{op}} e^{i 2 k_{1} a_{\mathrm{op}}} \\
& f_{\mathrm{Lamb}} \simeq-\frac{1}{2} G_{L} e^{i \Phi_{L}} \bar{a} e^{-2\left(\pi-\theta_{L}\right) \beta_{L}} \\
& \times e^{i 2 k_{1} \bar{a}\left[\left(c_{1} / \bar{c}_{L}\right)\left(\pi-\theta_{L}\right)-\cos \theta_{L}\right]-i \pi / 2} F_{L} \\
& \times \sum_{m=0}^{\infty}(-1)^{m} e^{-2 \pi m \beta_{L}} e^{i 2 \pi m k_{1} \bar{a} c_{1} / \bar{c}_{L}} e^{-(1 / 2) \gamma^{2} \sigma_{r}^{2}},
\end{aligned}
$$

where

$$
\begin{aligned}
& \theta_{L}=\sin ^{-1}\left(c_{1} / c_{L}\right), \\
& G_{L} \simeq 8 \pi \beta_{L} c_{1} / c_{L}, \\
& \bar{c}_{L} / c_{1} \simeq k_{1} \bar{a} /\left(\alpha_{L}+1 / 2\right),
\end{aligned}
$$

and

$$
\begin{aligned}
\gamma= & k_{1}\left\{2\left[\left(c_{1} / \bar{c}_{L}\right)\left(\pi-\theta_{L}\right)-\cos \theta_{L}\right]+2 \pi m c_{1} / \bar{c}_{L}\right. \\
& \left.+B k_{1} \bar{a}\left[2\left(\pi-\theta_{L}\right)+2 \pi m\right]\right\} .
\end{aligned}
$$

The terms $f_{\text {spec }}$ and $f_{\text {op }}$ contain a reflection coefficient $\mathcal{R}_{12}$ due to the interface they are associated with. Similarly, $f_{\text {Lamb }}$ contains a coupling coefficient $G_{L}$ that describes the efficiency with which the incident signal couples with the shell and reradiates. $B$ accounts, in part, for dispersion according to the relation $c_{1} / c_{L}=c_{1} / \bar{c}_{L}+B k_{1} \Delta_{a}$, where the mean speed $\bar{c}_{L}$ is $c_{L}$ evaluated at $k_{1} \bar{a}$. The terms $a_{\text {spec }}$ and $a_{\mathrm{op}}$ in Eqs. (7) and (8) for $f_{\text {spec }}$ and $f_{\text {op }}$ are the local radii of curvature that the incident wave sees, respectively. The $\bar{a}$ in Eq. (9) for $f_{\text {Lamb }}$ is the average value of the radius of the irregular sphere. The ray paths along lines of constant meridional angle experienced deviations in path length due to deviations in effective radius from this mean. The attenuation coefficient $\beta_{L}$ and dispersion term $\alpha_{L}$ of the Lamb wave are the imaginary and real parts of the complex root of the denominator of the modal-series coefficient for a fluid-filled elastic spherical shell. The term $\theta_{L}$ is the angle at which the Lamb wave launches and lands at the surface and $\Phi_{L}$ is an empirically determined phase shift due to the irregularity of the body. The term $\sigma_{r}$ is the root-mean-square (rms) deviation of the shell radius from the mean value $\bar{a}$. Note that there are several " $a$ " terms defined in the attempt to describe the irregularity of the shell. Although they differ in value, they are all comparable to $a_{\text {esr }}$.
Details of the above ray-based equations are discussed in Stanton et al. (1998b). One key aspect of this equation is the roughness-induced attenuation of the Lamb wave that was derived in that paper. In addition to the derived terms, there are empirically determined terms such as $F_{\text {spec }}, F_{\text {op }}$, and $F_{L}$ which are used to weight the different scattered rays. For example, the opercular opening may be aimed away from the acoustic system, preventing a ray from traveling into the opening and back out toward the system. In that case, $F_{\mathrm{op}}=0$. For the case when the opening is aimed toward the system, then $F_{\text {op }}$ is near or equal to unity. $F_{\text {spec }}$ and $F_{L}$ are also used to compensate the energy associated with the specular and Lamb waves with respect to the direction of the opercular opening.

The above ray formula presented in Eq. (6) is only valid for $k_{1} a_{\text {esr }}$ greater than unity. However, in that region it has the potential for predicting the resonance structure at least qualitatively, if not also quantitatively. The formula is useful for both single realizations and averages over ensembles.

\section{EXPERIMENTAL SETUP AND ANIMALS}

There has been a series of acoustic scattering experiments involving both planktonic and benthic shelled animals. The experiments involving the planktonic gastropods (2mm-long Limacina retroversa) were conducted in a laboratory-style tank setup on the deck of a ship using freshly caught live animals. The work conducted during two cruises (1993 and 1994), is described in Stanton et al. (1994, 1996, 1998a, and 1998b) and Chu and Stanton (1998). The recently completed experiments involving the benthic gastropods, periwinkles (Littorina littorea), were conducted during 1997 in a similar tank setup in a laboratory at the Woods Hole Oceanographic Institution. Because of the dominance of the shell in the scattering, the tissue of the benthic animals was removed (for experimental convenience) and the experiments were performed on the empty shells. Descriptions of the periwinkles and experimental setup are given below.

\section{A. Animals}

The periwinkles were collected during low tide off of rocks at the shoreline at Woods Hole, MA. This species was chosen, in part, because of its similarity in morphology to the planktonic gastropods studied earlier so that comparison of the scattering by the two different types of animals could be made. The periwinkle was also chosen because of the fact that this is a common shape of many benthic species. Studying this particular species could provide insight into the scattering by other ones of similar morphology.

The shape of both types of animals is in the form of a spiral (Fig. 1). More precisely, each animal is in the shape of a tube that is wound into a spiral. Each $360^{\circ}$ rotation of the tube corresponds to a cycle of growth. The material properties of the shell of each type of animal are very similar to each other-the planktonic shell is composed of aragonite (Lalli and Gilmer, 1989) and the benthic is a combination of aragonite and calcite (Lowenstam, 1954). Aragonite and calcite are similar materials. Both minerals are composed of $\mathrm{CaCO}_{3}$ (calcium carbonate) with their differences being in 
their crystalline structure. Also, the density, compressional sound speed, and shear sound speed of calcite are $2.71 \mathrm{~g} / \mathrm{cc}$, $6530 \mathrm{~m} / \mathrm{s}$, and $3360 \mathrm{~m} / \mathrm{s}$, respectively, while the density and compressional sound speed of aragonite are $2.92 \mathrm{~g} / \mathrm{cc}$ and $5820 \mathrm{~m} / \mathrm{s}$, respectively (the value of shear sound speed could not be found) (Carmichael, 1982). The differences in material properties will result in differences in scattering resonances for a given size and shape, but not overall scattering levels. The major acoustical difference between the two types of animals is due to the fact that the planktonic animals typically have much thinner shells than those of the benthic animals of the same size. The thinner shell allows the planktonic animal to swim throughout the water column.

Six periwinkles were used in the backscatter experiments ranging in size from 6.2 to $13.7 \mathrm{~mm}$ (Table I). The ratio of length to width of each animal is roughly constant for the range of sizes used. This is an important observation as it allows the use of a single shape for scattering predictions over a range of lengths. This quality of constant proportions involving outer dimensions as well as shell thickness is further illustrated in the studies involving a larger range of size of animals (Figs. 2 and 3).

\section{B. Experimental setup}

The experimental setup and associated aspects of system calibration and data acquisition are very similar to those described in detail in earlier papers (Stanton, 1990; Chu et al., 1992) and summarized in more recent papers (Stanton et al., 1994, 1998a) and will only be briefly summarized here. The principal difference between this setup and previously reported ones is that the acoustic transducers were aimed horizontally rather than being mounted on the bottom and looking up (Fig. 4). They were aimed in this direction to facilitate measurements of backscatter versus angle of orientation by use of a computer-controlled stepper motor (also a new addition) that rotated the shells suspended in the acoustic beam.

The system consists of an array of pairs of acoustic transducers that was facing in the horizontal direction in a 3.7 -m-long by $2.4-\mathrm{m}$-wide by 1.5 -m-deep tank. The tank was filled with filtered seawater. The transducers within each pair are closely spaced and identical. One of the two transducers is used as the transmitter and the other the receiver. By using two transducers, closer scattering ranges can be achieved, ringing of the transmitter is less of a problem, the system is linear, and the system is easy to calibrate. The transducers span the frequency range of $24 \mathrm{kHz}$ to $1 \mathrm{MHz}$ (although note that the $24-\mathrm{kHz}$ transducers, new additions, are physically separate from the array). The frequencies used in this particular experiment were 24, 50, 75, 120, 165, 200, 250 (BB), $500 \mathrm{kHz}(\mathrm{BB})$, and $1 \mathrm{MHz}(\mathrm{BB})$ where broadband (BB) octave-bandwidth transducers were used in the upper frequency region. The transducers at frequencies of $200 \mathrm{kHz}$ and below were narrow band.

The pulse-echo electronics were controlled by the same personal computer that controlled the stepper motor and included a programmable waveform generator and power amplifier at the transmission end of the system and a preamplifier, bandpass filter, and digital oscilloscope (for capture,

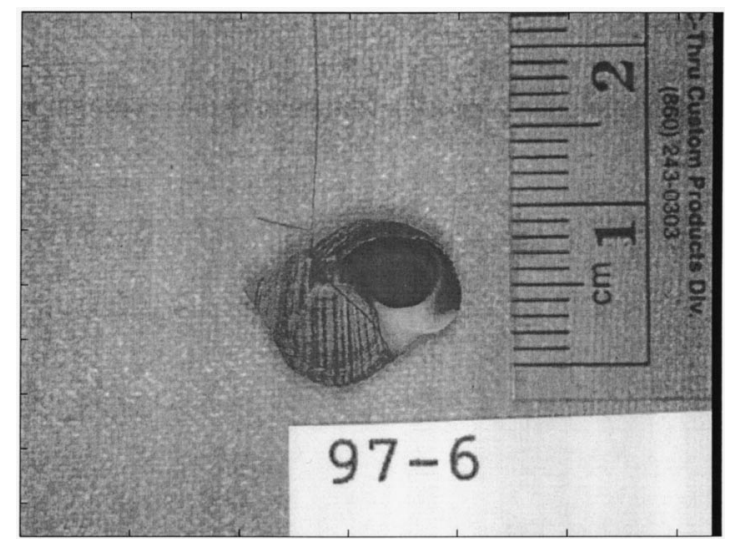

FIG. 1. Photo of periwinkle (Littorina littorea, animal No. 97-6) used in the 1997 acoustic scattering experiments at Woods Hole Oceanographic Institution. This is close to what would be a side view in the scattering measurements. The thin line is the tether ( $59 \mu \mathrm{m}$ diameter). The opercular opening is facing the camera similarly to how it faced the acoustic transceiver at an orientation of about $240^{\circ}$.

display, and transfer of data to computer) at the receiving end of the system.

The animals were suspended in the middle of the acoustic beams by a single thin monofilament line that was $59 \mu \mathrm{m}$ in diameter and was acoustically transparent at these frequencies. Each animal was at a range of $51 \mathrm{~cm}$ from the transducer pairs spanning $50 \mathrm{kHz}-1 \mathrm{MHz}$ and at a range 73 $\mathrm{cm}$ from the $24 \mathrm{kHz}$ transducers. These distances correspond to the far field of each transducer. Also, at this range, each animal was within the first Fresnel zone of the transceiver, making this a far-field scattering condition. The line was tied around the midsection of the body (Fig. 1) and no glue was used (the tying of the tether onto these small shells was done by first affixing the shell onto a piece of two-sided tape, then using tweezers to wrap the tether around the shell and tie the knots). The tether was tied to a clip that was connected to the shaft of the stepper motor so that the tether coincided with the center of the shaft. The shells were heavy enough so that the tether was fully extended throughout the experiment. Through use of the small torsional strength of the tether,

TABLE I. Outer dimensions and related quantities of the periwinkles used in the acoustic scattering measurements. The length is the measured maximum tip-to-tip distance. The width was measured between the plane containing the face of the opercular opening and the outer point of the shell on the opposite side of the shell. Because of the complexity of the animal shape and in order to be consistent, the axis along which the width was measured was not necessarily perpendicular to the axis along which the length was measured. The equivalent spherical radius $a_{\mathrm{esr}}$ is used for some simulations and is the radius of the sphere that has the same volume as that of the animal. The volume of the animal is calculated by assuming it has a prolate spheroidal shape with length and width as given in this table.

\begin{tabular}{ccccc}
\hline \hline $\begin{array}{c}\text { Animal } \\
\text { ID number }\end{array}$ & $\begin{array}{c}\text { Length } \\
(\mathrm{mm})\end{array}$ & $\begin{array}{c}\text { Width } \\
(\mathrm{mm})\end{array}$ & $\begin{array}{c}a_{\text {esr }} \\
(\mathrm{mm})\end{array}$ & Length/width \\
\hline $97-1$ & 6.2 & 3.9 & 2.3 & 1.6 \\
$97-2$ & 6.6 & 4.2 & 2.4 & 1.6 \\
$97-3$ & 8.8 & 5.6 & 3.3 & 1.6 \\
$97-4$ & 10.0 & 6.7 & 3.8 & 1.5 \\
$97-5$ & 10.6 & 7.0 & 4.0 & 1.5 \\
$97-6$ & 13.7 & 8.4 & 4.9 & 1.6 \\
\hline \hline
\end{tabular}




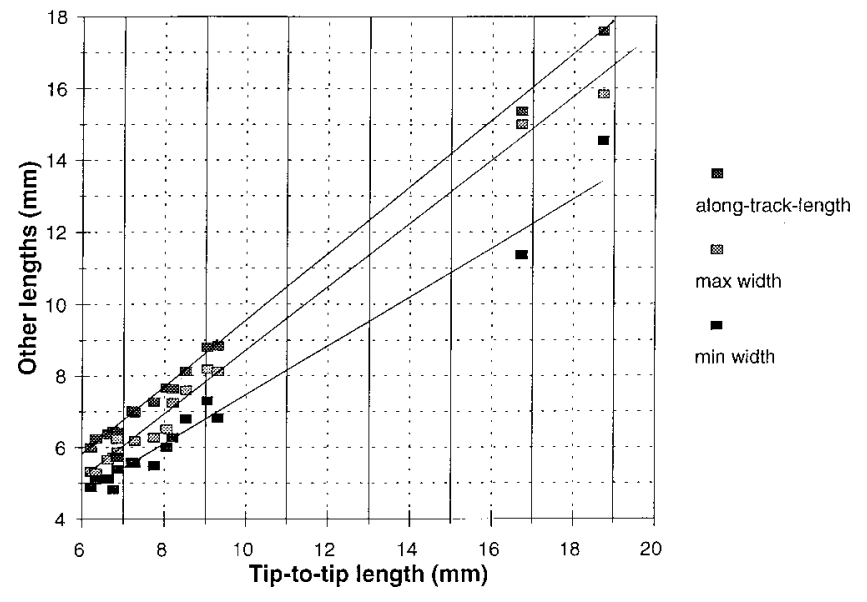

FIG. 2. Measurements of several different outer dimensions of various sized periwinkles plotted as a function of maximum tip-to-tip length. These were different animals (but same species caught at the same time and location) than the ones used in the acoustics experiment. The along-track length is the length of the animal (very close to the maximum length) along the track of the acoustic signal when aimed at the apex (i.e., end-on incidence). The widths were measured generally transversely with respect to the lengthwise axis. Given the complexity of the shape of the body, the crosswise axes weren't necessarily perpendicular to the lengthwise axis, although they were nearly so. The lines are least-square fits.

rotation of the stepper motor shaft could cause the shell to rotate the same amount (difficulties with this approach are discussed in Sec. IIC).

\section{Measurement procedure}

The system was calibrated both before and after the entire series of scattering measurements (i.e., involving all periwinkles) was performed. Each scattering measurement involved a sequence of first measuring the background reverberation of the tank without the animal present in the beam, then placing the animal in the beam and measuring the backscatter over the range of orientation angles $0^{\circ}-450^{\circ}$ in

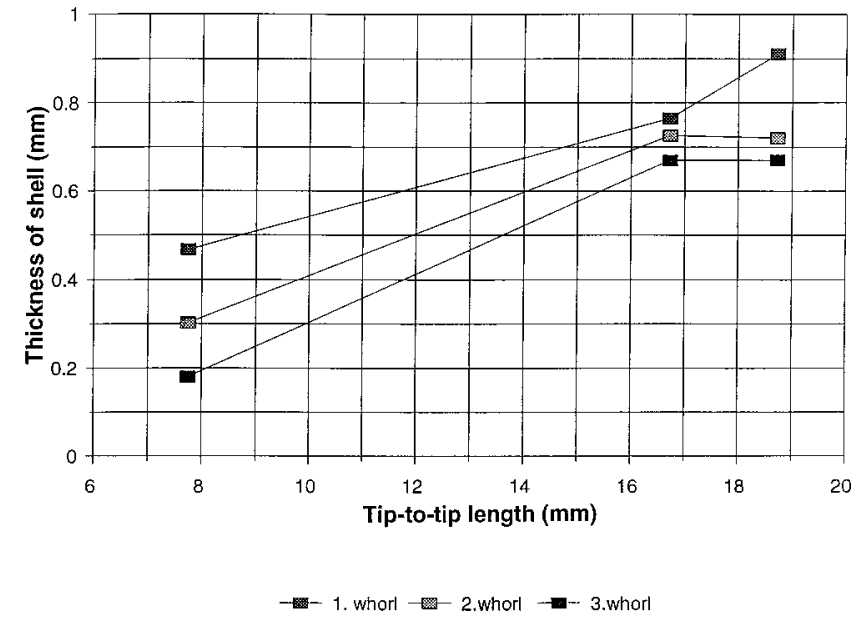

FIG. 3. Thickness of animal shell plotted as a function of tip-to-tip length of body. Because of the growth process of the animal, the shell was naturally of varying thickness (the older sections of the shell were thicker). Therefore, thickness as measured from several different sections or "whorls" within each body are plotted. Each whorl corresponds to one $360^{\circ}$ rotation of the spiral shape of the body, which corresponds to one growth cycle. The apex of the shell is solid. The lines drawn were simply to connect data from the same class of whorl.

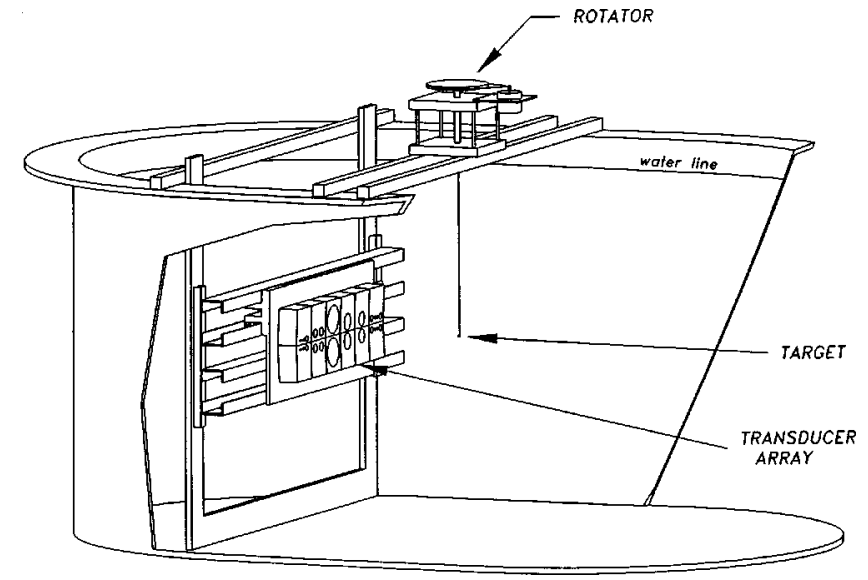

FIG. 4. Experimental setup for measurements of acoustic backscatter versus angle of orientation.

as small as $1^{\circ}$-increments. The scattering measurements were performed using each of the transducers listed above with one animal (No. 97-1) and using a subset of the transducers for the other five animals.

Calibration was performed by configuring the transducer elements so that the transmitter and receiver transducers of each pair were separated and facing each other. The separation distance was $198 \mathrm{~cm}$ for the $24 \mathrm{kHz}$ transducers and 68 $\mathrm{cm}$ for all other frequencies. Separation was done by first placing the entire array on the bottom of the tank and then raising each subarray (i.e., the linear array that contains one transducer from each pair) so that the axis of each subarray is aligned vertically and the transducers are facing in the horizontal direction.

Since bubbles can greatly contaminate the quality of the measurements, great care was taken so that there were no bubbles present. Before calibration and the backscatter measurements, all transducers were soaped so that their surfaces would be in good contact with the water and buildup of bubbles would be retarded. Furthermore, the animal shells and tether were soaped before the backscatter measurements. Soaping the shells involved soaking them in soapy water and agitating them so that the soap could reach the interior.

In addition to the initial soaping, the tether was occasionally wiped so that any bubbles that may be building up but were not detected visually could be eliminated. Also, before each time a new shell was placed in the beam for a scattering measurement, a high-speed jet of water was aimed into the opercular opening so that bubbles and debris would be removed from the interior of the shell. The jet, which was produced by a laboratory fluid dispenser, was repeatedly pulsed for several minutes to insure that no bubbles remained inside the shell.

During the background reverberation measurements, the echoes were summed over many pings so that random noise would be eliminated and the resultant coherent echo was stored in the digital oscilloscope. Once the animal was placed in the acoustic beam for the animal scattering measurements, the oscilloscope subtracted the background signal from the echo in real time so that all that remained was the echo from the animal (plus random noise). This procedure was especially important for the lower frequencies where the 
reverberation from various parts of the tank dominated the echoes. Because of the low signal-to-noise ratio for the 24$\mathrm{kHz}$ measurements, the animal echo after subtraction was also summed from repeated transmissions so that the random noise in the echo could be reduced. The $50-\mathrm{kHz}$ backscatter measurements would not have been possible without the subtraction procedure, and the $24-\mathrm{kHz}$ measurements required both procedures.

During the backscatter measurements, great care was taken so that all animals were rotated through the same body plane and that the set of angles of rotation from each animal was the same (e.g., $0^{\circ}$ was the same orientation relative to the transducers for each animal). The lengthwise axis of each animal was approximately horizontal. In order to align the animals in a consistent manner, the shaft was rotated manually immediately before the measurements began for a given animal so that each experiment would begin with the apex of the animal facing the transducer pair. More precisely, the animal was aligned so that the line connecting the outer tip of the opercular opening (opposite the apex) and the apex intersected the middle of the transducer pair. The opercular opening was aimed in the horizontal direction so that at approximately $240^{\circ}$ of rotation, the opening would be facing the transducer. The alignment was performed visually and showed excellent repeatability. By use of the computer control, the scattering measurement involved a sequence of alternating single pings and rotations of $1-3^{\circ}$ of the stepper motor.

One challenge involved the fact that there appeared to be torsional friction on the tether used to suspend the animals. The source of this friction is perhaps from the surface tension of the water. Because of this friction, the rotation of the animal didn't always correspond with the rotation of the stepper motor. In order to insure one-for-one correspondence between the rotation of the motor and animal, all experiments involved $450^{\circ}$ of rotation. After each run, the polar plots of backscatter versus angle of orientation were visually checked. If there was not good agreement between the $0-90^{\circ}$ data and the $360-450^{\circ}$ data, the data set was rejected and the measurement was repeated. Sometimes the measurement needed to be repeated several times to produce satisfactory results. The basis for acceptance was straightforward. If any slippage due to the friction occurred, there would always be a substantial amount of it. Thus the rejected data sets would have slippage of the order of tens of degrees and would be obviously unacceptable visually. The acceptable data sets had a very close agreement between the $0-90^{\circ}$ data and $360-450^{\circ}$ data, as will be illustrated in the next section.

\section{EXPERIMENTAL RESULTS}

The acoustic backscatter data were analyzed in both the frequency and time domain as well as with respect to animal orientation. In the frequency domain, the target strength versus frequency is investigated for single pings at a fixed angle of orientation as well as averaged over a range of angles. In the time domain, the broadband signals are compressed via a cross-correlation method that resembles matched filtering.
This temporal analysis, which is used in concert with the spectral analysis, is crucial in understanding the dominant scattering mechanisms.

\section{A. Observations of dependence of scattering upon acoustic frequency, animal orientation, and animal size}

There were strong dependencies observed of the acoustic backscattering upon acoustic frequency as well as size and orientation of the animals (Figs. 5-8). These dependencies are illustrated in plots of backscattering versus orientation at various fixed frequencies or bands of frequencies and fixed animal size (Figs. 5, 6); backscattering versus orientation at fixed frequency and different animal sizes (Fig. 7); and backscattering versus frequency at various fixed angles of orientations (Fig. 8).

Except for the lowest frequencies, the backscattering by the periwinkles was strongly dependent upon angle of orientation (Fig. 5). The orientation dependence also varied strongly with frequency. Generally, the data at the lowest frequencies showed that the backscattering was mildly dependent upon orientation. As frequency is increased, the lobes of the scatter pattern tend to become narrower as well as greater in number.

At the higher frequencies, there was much structure in the scatter patterns. In an attempt to eliminate much of the fine structure that was highly variable from frequency to frequency, the directivity patterns were investigated after an average across the band of frequencies from each of the broadband transducers was made (Fig. 6). Some of the fine structure was, indeed, eliminated, although some strong sidelobes still remained.

For a fixed frequency, the orientation dependence of the backscattering also varied with animal size (Fig. 7). At 50 $\mathrm{kHz}$, the data show that the scatter pattern is nearly omnidirectional for the smallest animal and strongly directional for the largest animal.

At any given fixed angle of orientation of the animal, there is also significant dependence of the backscattering upon acoustic frequency (Fig. 8). At each angle over the entire range of orientations, there existed a very strong structure containing peaks and deep nulls. The peaks and nulls were generally not regularly spaced.

\section{B. Compression of broadband echoes in time domain}

In addition to the spectral analysis described above, the echoes from an individual periwinkle were analyzed in the time domain as well. All transmitted waveforms were sufficiently long (usually $200-400 \mu$ s) that individual features could not be resolved in the time domain without further processing. Because of the broadband nature of the echoes made possible by the octave-bandwidth transducers, the echoes using those transducers could be temporally compressed so that some of the features could be revealed.

The compression was performed by cross-correlating the echoes with the waveform received in the calibration. This "replicate" corresponded to the echo one would expect in the backscatter measurement if a target possessing a uniform 

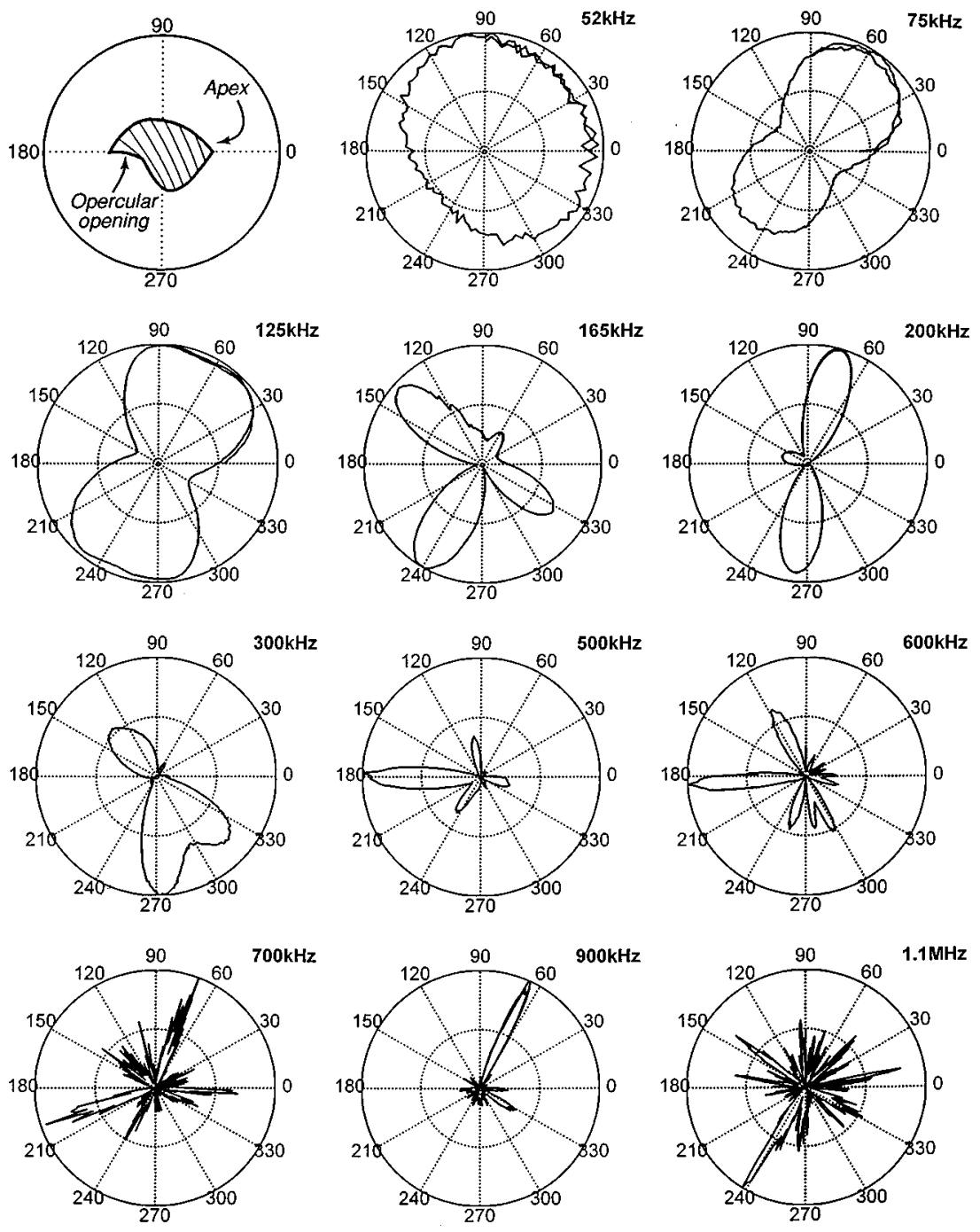

FIG. 5. Normalized differential backscattering cross section $\sigma_{b s}$ (linear scale, not logarithmic) versus angle of orientation for a number of frequencies for an individual periwinkle animal (No. 97-1, 6.2-mm long). Each plot was normalized by its maximum value, resulting in a $0-1$ range of normalized $\sigma_{b s}$. Bottom view of animal in experiment is sketched in upper left corner. The data were collected over the range of angles 0 to $450^{\circ}$ in $1^{\circ}-3^{\circ}$ steps $\left(1^{\circ}\right.$ steps for frequencies at or above $165 \mathrm{kHz}, 2-3^{\circ}$ steps for the lower frequencies). Data from all $450^{\circ}$ are shown in order to illustrate reproducibility of the rotation of the animal. $0^{\circ}$ corresponds to the apex of the shell aimed at the acoustic transducers (the apex was actually aimed slightly above the transducers - an amount that varied from animal to animal). The opercular opening faces the transducers at approximately $240^{\circ}$ (the normal of the plane of the opening faced slightly above the transducers at $240^{\circ}$ ). The overlap between the main lobe at about $65^{\circ}$ in the $900-\mathrm{kHz}$ data that was observed in both the $0-90^{\circ}$ data and in the $360-450^{\circ}$ data illustrates the high degree of reproducibility with this tether arrangement. The $24-\mathrm{kHz}$ data for this animal was only collected at $0^{\circ}$ and is therefore not shown. $k_{1} a_{\mathrm{esr}}$ $=1$ at about $100 \mathrm{kHz}$ for this animal.
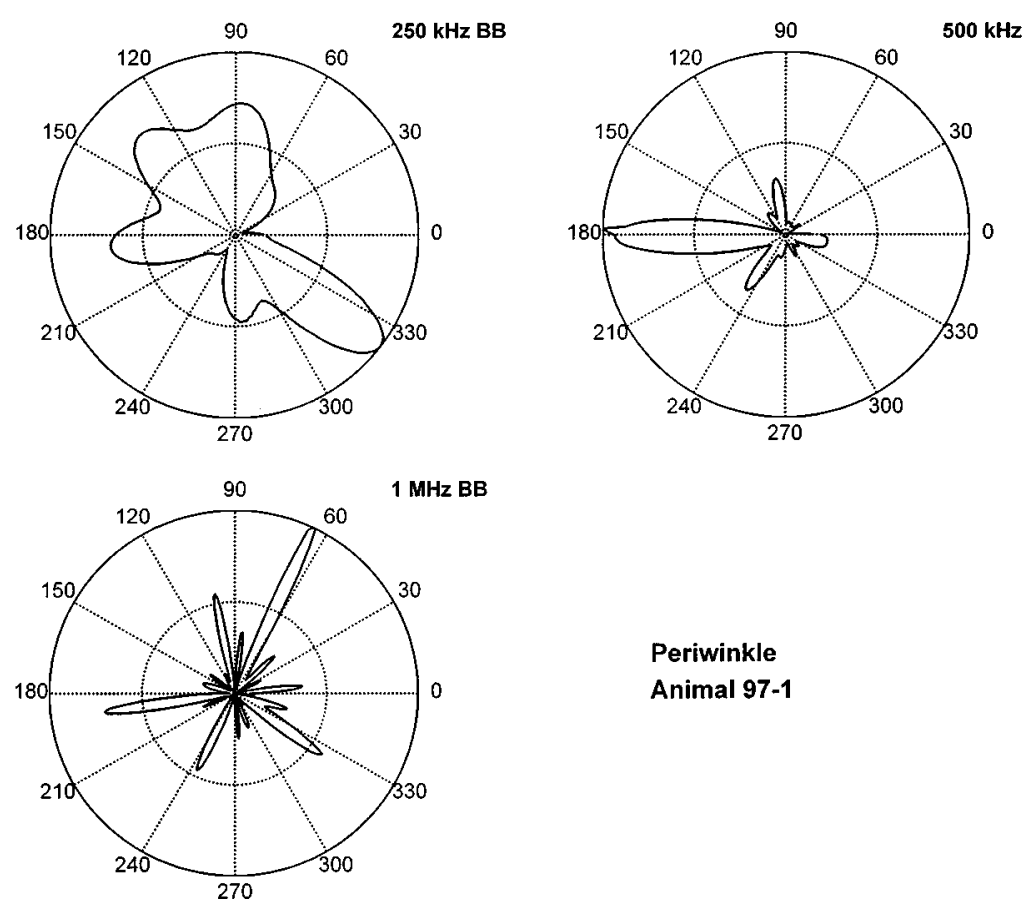

Periwinkle

Animal 97-1 

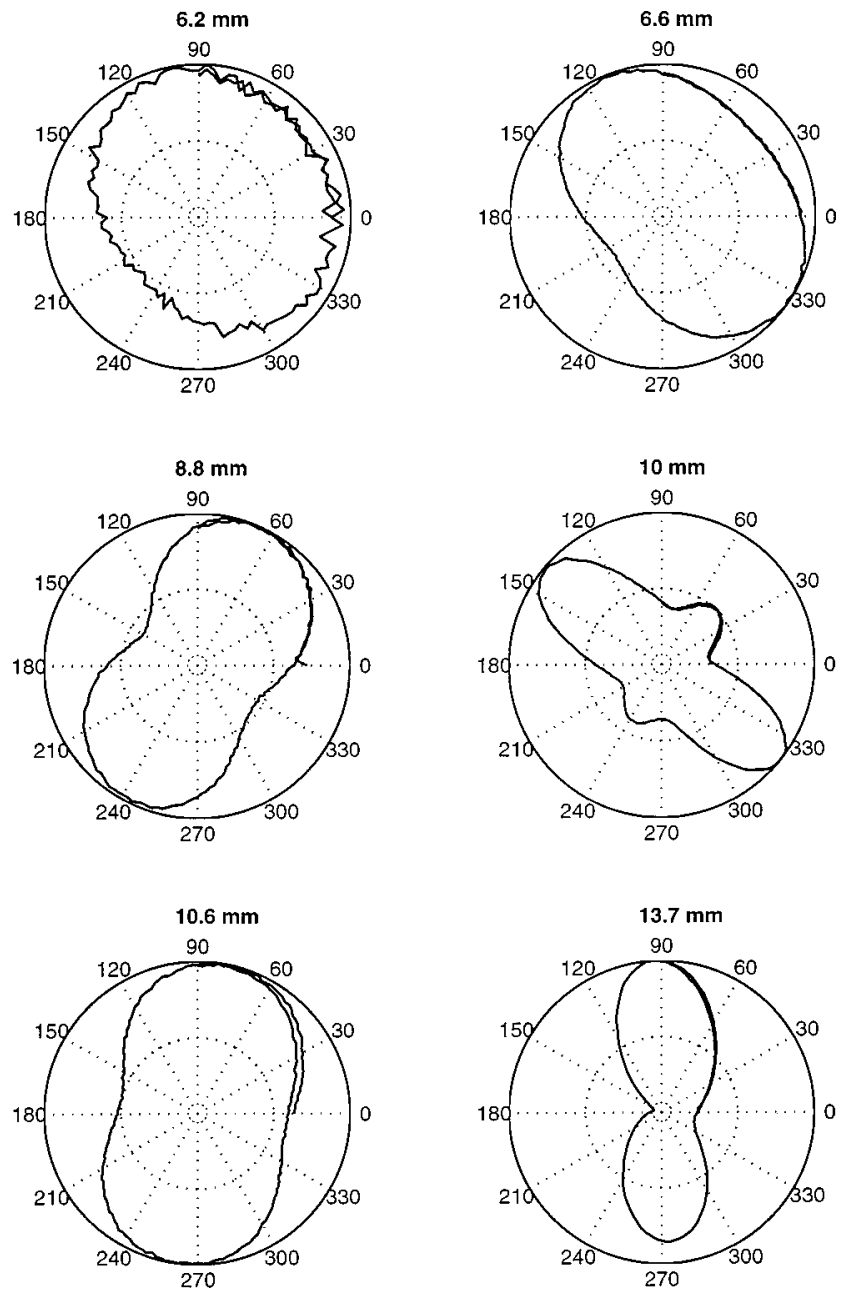

FIG. 7. Normalized differential backscattering cross section $\sigma_{b s}$ (linear scale, not logarithmic) versus angle of orientation for six individual periwinkles of different sizes at $50 \mathrm{kHz}$. The animals ranged in length from 6.2 to $13.7 \mathrm{~mm} . k_{1} a_{\text {esr }} \cong 1$ for the $13.7-\mathrm{mm}$-long animal. Each plot was normalized by its maximum value, resulting in a $0-1$ range of normalized $\sigma_{b s}$. Data collected in same manner as described in Fig. 5 caption. frequency response was used. This cross-correlation process is formally known as a matched filter if the replicate incorporates the scattering properties of the target. Our replicate does not incorporate the scattering properties since it is those properties that are unknown and under investigation. Therefore, the pulse-compression process performed on these echoes generally was not a matched filter. However, the deviations of the cross-correlation process using the idealized (uniform frequency response) replicate from what one would expect from a matched filter output contains information (Stanton et al., 1998a; Chu and Stanton, 1998). For example, if the scatterer is composed of a number of facets, the compressed pulse output using the calibration waveform for the replicate as described above will be composed of multiple main lobes, one per feature.

The compressed pulse output for the 500-kHz broadband data from animal 97-1 showed a structure in the (compressed) time series that depended upon orientation (Figs. 9 and 10, Table II). For many orientation angles, the output contained two or more major lobes. The separation or time delay between the lobes varied with angle. Some of the time delays corresponded to distances greater than what one would expect from two facets positioned along the direction of propagation of the signal and separated by a distance equal to the diameter of the body. Thus it is apparent that more than a simple facet or feature-based model is required to describe the scattering. These latter features are qualitatively consistent with those subsonic circumferential waves observed with planktonic gastropods (Limacina retroversa) in Stanton et al. (1998a, b) and Chu and Stanton (1998).

\section{COMPARISON OF SCATTERING PREDICTIONS WITH DATA}

The structure in the scattering data (plotted versus frequency) presented in Sec. III indicates that there are interfer-
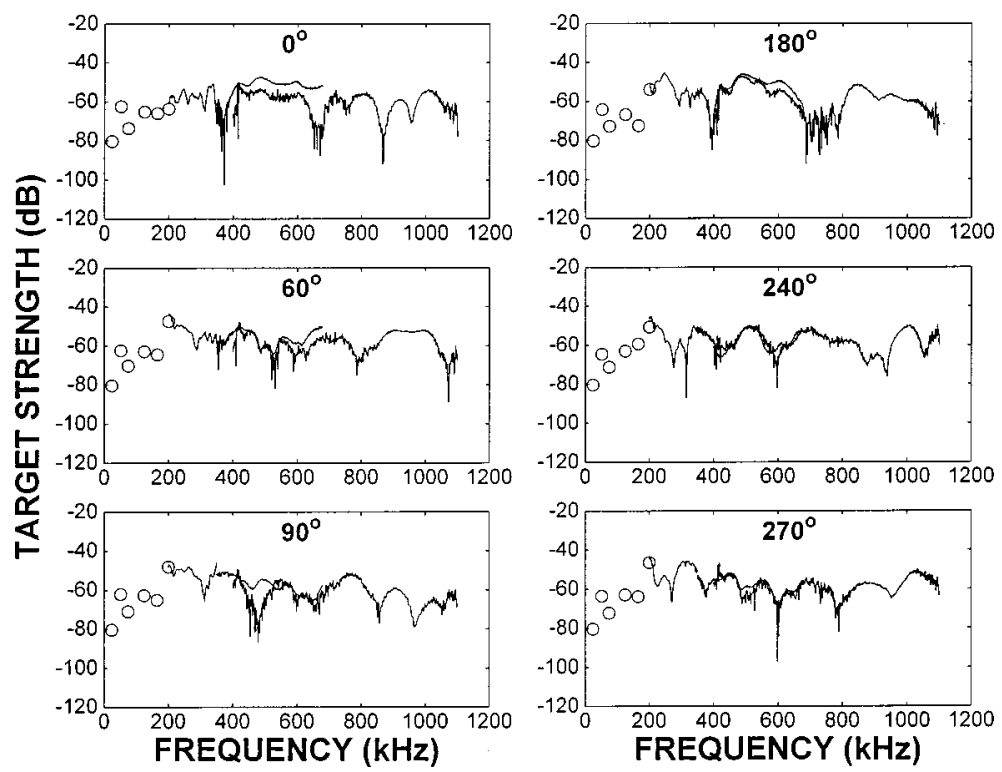

FIG. 8. Target strength versus frequency for six angles of orientation for an individual periwinkle (No. 97-1, $6.2-\mathrm{mm}$ long). Four of the angles were chosen arbitrarily as they were multiples of $90^{\circ}$. The angles 60 and $240^{\circ}$ were chosen because they corresponded to peaks in some of the scattering patterns, as illustrated in Fig. 5. $k_{1} a_{\mathrm{esr}}=1$ at about $100 \mathrm{kHz}$ for this animal. The discrete points are data from single-frequency transducers while the continuous curves are data from the broadband transducers. The overlapping curves are due to the fact that frequency ranges of the broadband transducers sometimes overlapped each other. Data collected in same manner as described in Fig. 5 caption. Since the scattering pattern was essentially uniform with respect to angle for the $24 \mathrm{kHz}$ measurement, the TS value for $24 \mathrm{kHz}$ made at $0^{\circ}$ was used in each plot for that frequency. 


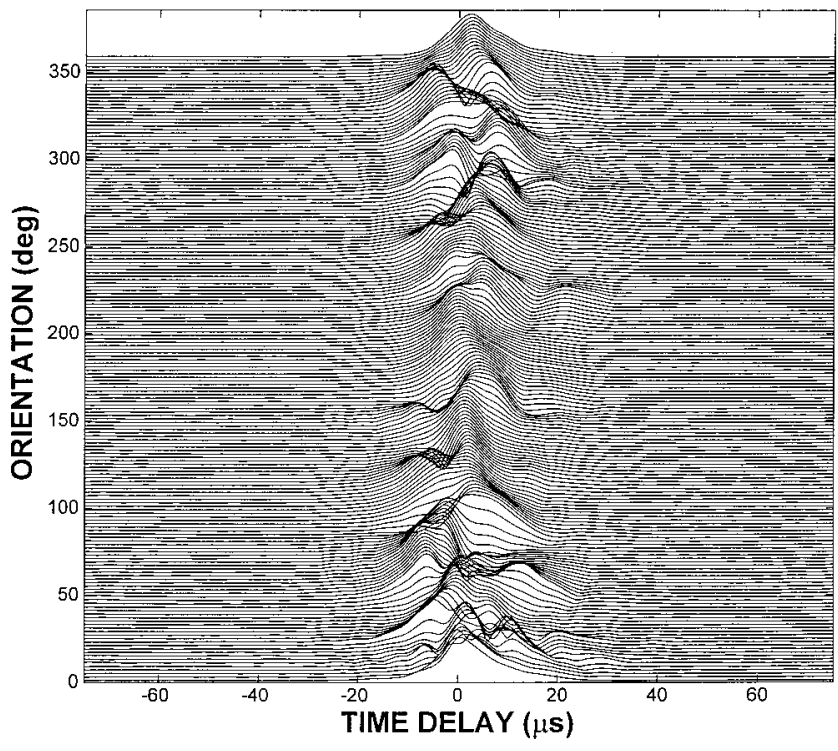

FIG. 9. Compressed pulse (CP) output for 500-kHz broadband echoes from individual periwinkle (animal 97-1, 6.2-mm long) as a function of orientation angle. Data collected as described in Fig. 5 caption. For the purposes of illustration, only a subset of the angles over the entire range 0 to $360^{\circ}$ is shown. Each CP output is normalized to the same peak amplitude.

ence phenomena taking place. These phenomena are most likely due to multiple rays of different phases being scattered back toward the receiver and interfering with each other. The presence of multiple returns in the compressed pulse data is consistent with this hypothesis. The dependence of scattering versus angle of orientation of the animal is consistent with the fact that the shape of the shell is complex. The amplitude and phase of the various classes of scattered rays are most likely changing with orientation.

The structure of the scattering for high $k_{1} a_{\text {esr }}$ is modeled on a ping-by-ping basis using a ray-based approach. In order to estimate the scattering over all $k_{1} a_{\text {esr }}$, a modal-seriesbased approach is used, but only over ensemble-averaged echoes. In all predictions, the material properties for calcite are used as an approximation to the shell material. As discussed earlier, the shell is composed of two very similar materials - aragonite and calcite. To the authors' knowledge, complete information on material properties is only available on calcite, but not aragonite or calcite-aragonite. Given the closeness of (known) material properties of calcite and aragonite, use of calcite-only parameters is a reasonable approximation.

\section{A. Single-ping analysis}

The structure of the patterns in the plots of target strength versus frequency for high $k_{1} a_{\text {esr }}$ is not predictable using the exact modal-series solution to the elastic fluidfilled spherical shell. Given the complexity of the shape of the animal, this is not a surprise. In order to model the structure of the scattering by these complex shapes, an approximate ray approach is used. This approach is intuitive and physically tractable to various rays that will scatter geometrically and cause interference such as the ones observed in Fig.

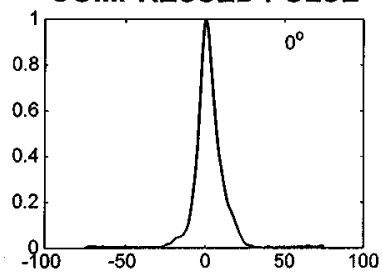

TARGET STRENGTH
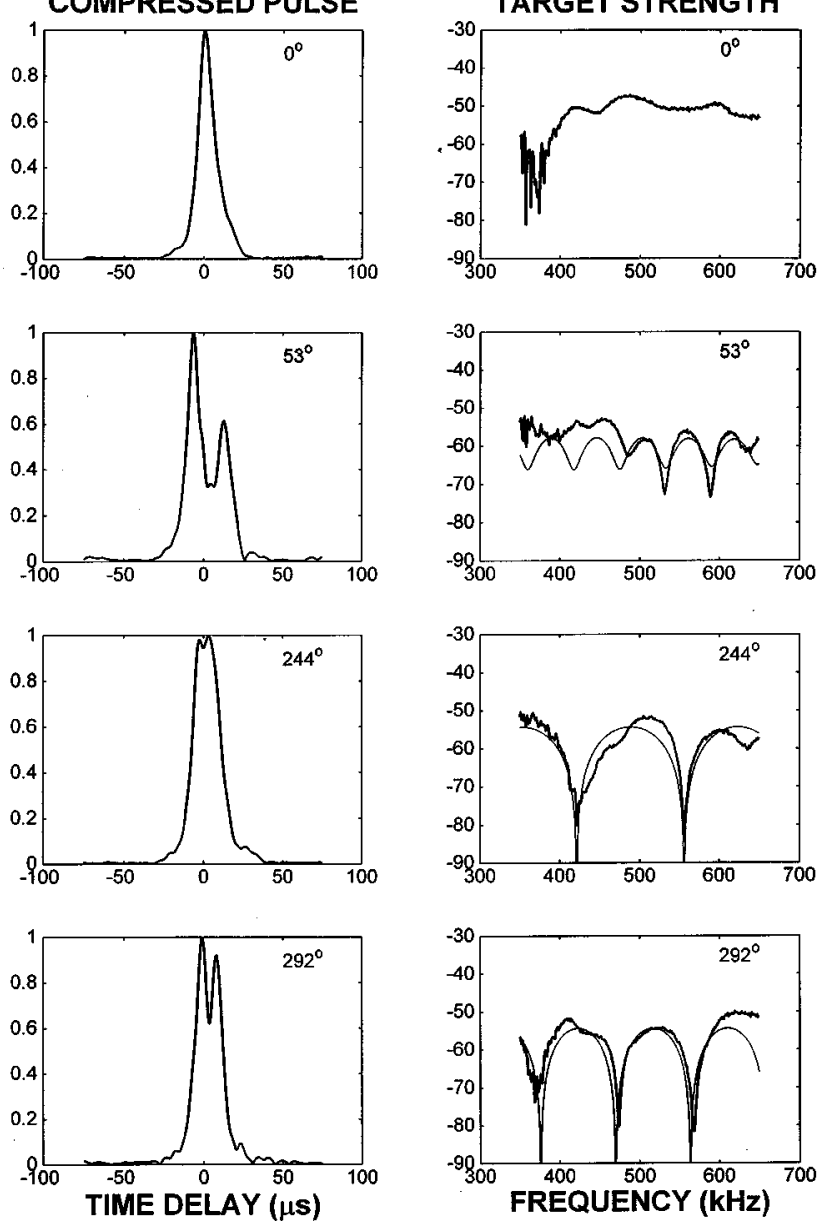

FIG. 10. Compressed pulse (normalized), frequency spectrum, and raybased scattering predictions for several orientations of an individual periwinkle (animal No. 97-1, 6.2-mm long). Data (thick lines) from set described in Fig. 5. Theoretical predictions (thin lines) were produced as follows: $53^{\circ}$ orientation: These calculations were similar to those for Limacina retroversa given in Fig. 2 of Stanton et al. (1998b) with the main difference being animal size, $F_{\text {spec }}$, and Lamb wave speed. Equation (6) of this paper was used with only $f_{\text {spec }}$ and $f_{\text {Lamb }}$ contributing $\left(f_{\text {op }}=0\right)$. $\mathcal{R}_{12}$ $=0.84, a_{\mathrm{spec}}=\bar{a}=a_{\mathrm{esr}}=2.28 \mathrm{~mm}, \sigma_{r}=0.025 \bar{a}$ (inferred from the data), $\beta_{L}$ $=0.002 k_{1} \bar{a}$ (the $k_{1} \bar{a}$ functional dependence is based on the analytical Lamb wave model and the coefficient 0.002 is based upon a fit to the data), $\alpha_{L}$ $=3 k_{1} \bar{a}$ (the " 3 ", is based on fit to data; the " $k_{1} \bar{a}$ ', is based on model), $\Phi_{L}=-\pi / 2$ (inferred from data) for the subsonic wave, $\theta_{L}=\pi / 2$ (based on model), $c_{L} / c_{1}=k_{1} \bar{a} /\left(\alpha_{L}+0.5\right) \simeq 1 / 3$ (this analytical relationship was predicted theoretically and the value of $1 / 3$ was observed), $F_{L}=1$ (inferred), $F_{\text {spec }}=1$ (inferred), (the $\mathcal{R}_{12}=0.84$ is calculated for the coefficient expected for a semi-infinite planar half-space of calcite where the published values for calcite $g \equiv \rho_{2} / \rho_{1}=2.646$ and $h \equiv c_{2} / c_{1}=4.345$ (longitudinal waves) (from Carmichael, 1982), the Lamb wave series was truncated to include only the $m=0$ term, and $F_{\text {op }}=0$. For the 244 and $292^{\circ}$ orientations: Equation (6) of this paper was used with $f_{\text {spec }}$ and $f_{\text {op }}$ contributing $\left(f_{\text {Lamb }}=0\right)$. For both cases, $g$ and $h$ are the same as for the $53^{\circ}$ orientation above, $F_{\text {spec }}=1$, $F_{\text {Lamb }}=0$, and the term $a_{\text {spec }}=a_{\text {op }}=a_{\text {esr }}=2.28 \mathrm{~mm}$ in the magnitude portion of each term. The phase shifts, which were more sensitive to shape and size, required adjustment from the mean radius for a good fit to the data: for $244^{\circ}$ orientation, $a_{\mathrm{spec}}=a_{\mathrm{op}}=1.22 a_{\mathrm{esr}}$ in the exponent and $F_{\mathrm{op}}=e^{-i \pi / 4}$; for $292^{\circ}$ orientation, $a_{\mathrm{spec}}=a_{\mathrm{op}}=1.75 a_{\mathrm{esr}}$ in the exponent and $F_{\mathrm{op}}=1$.

10. For some angles and bands of frequency, the interference pattern is weak (top row, Fig. 10), indicating that only one ray is dominating the scattering for that orientation. For other angles, two or more rays contribute to the scattering.

The raylike behavior of the scattering at the high $k_{1} a_{\text {esr }}$ 
TABLE II. Time separation between main features of compressed pulse output from individual periwinkle for certain angles of orientation. Data from Fig. 10. The time delay from the $53^{\circ}$ data is consistent with that of subsonic circumferential waves. The time delay from the $292^{\circ}$ data is consistent with the sonic wave round trip between two facets separated by a distance comparable to the size of the body. At 0 and $292^{\circ}$ orientation, the apex and opercular opening of the shell are aimed at, or in the general direction of, the transducer pair, respectively.

\begin{tabular}{ccl}
\hline \hline $\begin{array}{c}\text { Orientation } \\
(\text { degrees })\end{array}$ & $\begin{array}{c}\text { Time separation } \\
(\mu \mathrm{sec})\end{array}$ & \multicolumn{1}{c}{ Comments } \\
\hline 0 & $\ldots$ & single peak \\
53 & 19 & subsonic wave \\
292 & 9 & sonic wave \\
\hline
\end{tabular}

region is consistent with the type of scattering behavior observed with the limited set of data with the 2-mm-long planktonic gastropods (Limacina retroversa) in Stanton et al. (1998a, b). With those animals, there was an oscillatory behavior in the scattering that was dependent upon orientation. The oscillations were consistent with the presence of a subsonic Lamb wave with a speed of approximately $1 / 8$ that of the surrounding water.

In these periwinkle data, both a subsonic and sonic wave are observed after the initial echo and both have been modeled (Table II, Fig. 10). The data containing the sonic wave are consistent with rays scattering off of two facets where the path of the acoustic wave was in the water (bottom two rows of Fig. 10). The round-trip time is consistent with distances comparable to the diameter of the body. In this latter case, Eq. (6) was used, using Eqs. (7) and (8) for the specular (front interface) and opercular opening rays. The Lamb wave contribution from Eq. (9) was not included (i.e., $F_{L}=0$ ).

The data containing the subsonic wave indicate that there are two waves - one from a facet on the body (a likely candidate being the front interface) and the other due to the antisymmetric zeroth-order Lamb wave which is subsonic and travels around the elastic shell (second row of Fig. 10). Here, Eq. (6) was used with Eqs. (7) and (9) to include the specular and Lamb wave contributions, but excluding the opercular ray (i.e., $F_{\text {op }}=0$ ).

There is excellent comparison between the two-ray model and the data where the sonic waves are present. The orientations for these data correspond to ones where the opercular opening is facing in the general direction of the receiver. Hence it is reasonable to obtain two sonic waves, one from the front interface and one that travels into the opercular opening and back toward the receiver to interfere with the specular return.

For the data involving subsonic rays, there was reasonable agreement between the predictions and the data for the upper portion of the frequency band and poor agreement between the data and predictions for the low part of the band. The fact that there is agreement over only part of the band indicates that the scattering is more complex than modeled and other rays may exist that contribute significantly to the scattering. This is not a surprise given the simplifications leading up to the predictions. Only one of the large class of Lamb waves was included, and other waves were excluded as well.
What is remarkable about the portion of the frequency band in which the subsonic wave analysis is consistent with the data is the fact that the equation used was the same one used in the predictions of the scattering by the planktonic gastropods in Stanton et al. (1998b). In that analysis, a combination of measured parameters (e.g., animal size), inferred parameters (e.g., roughness-induced phase shift of Lamb wave), and parameters that were a compromise between inferred values and values obtainable through simplified theoretical predictions (e.g., Lamb wave coupling coefficient) were used. All of those parameters were used in this current analysis with the exception of animal size (the size of this periwinkle was used), $F_{\text {spec }}$ (which is expected to vary with orientation), and Lamb wave speed. The inferred subsonic wave was $1 / 3$ that of the sonic speed versus $1 / 8$ for the Limacina. This difference is to be expected since this set of data is at a higher $k_{1} a_{\text {esr }}$ value than for the Limacina as well as involving a disproportionately thicker shell, and the Lamb wave speed naturally increases with $k_{1} a_{\text {esr }}$ and relative shell thickness in that region (Kargl and Marston, 1989; Kaduchak et al., 1995).

This ray analysis illustrates some of the basic physics of the scattering process. At other angles, the structure is less regular, indicating that the scattering is more complex and would most likely require more rays in the predictions.

\section{B. Average echoes}

It is clear in the above single-ping analysis that the scattering process is very complex. It is so complex, that it may not be practical to be able to accurately predict the scattering for each angle of orientation. In field applications, observations of acoustic scattering usually involve aggregations of animals (both planktonic and benthic applications). These aggregations will involve a distribution of animal size and orientation whose ensemble-averaged levels will tend to have much less structure in the scattering pattern. Thus it is important to use a formulation that can, at the least, predict the averaged smoothed scattering levels.

One candidate for predicting the ensemble-averaged scattering is the exact modal-series solution for the fluidfilled elastic spherical shell. Although it cannot predict the structure of the scattering from single pings for these animals, it can be useful as an approximation in predicting the averaged scattering because it contains much of the underlying physics of the scattering process. Implicit in the solution are the specular wave, all classes of Lamb waves, internally transmitted waves, and Franz waves. Other waves, such as those entering the opercular opening, are not included. Also not included are effects of the irregularities of the shell on the various waves. However, by averaging the predictions over size and shell thickness for values of $k_{1} a_{\text {esr }}$ above 0.5, the resultant variation in phase of the (implicit) waves due to the different sizes and shell thicknesses will tend to have a similar effect that the irregularities have on the waves and smooth out the structure.

The averaging process consisted of averaging the backscattering cross section, as predicted by the exact modalseries solution, over a range of sizes and shell thicknesses. The averaging was only performed for values of $k_{1} a_{\text {esr }}$ above 
0.5 and only over a small range of $a_{\text {esr }}$ and shell thickness (the range of $a_{\text {esr }}$ and shell thickness corresponded to measured irregularity of the shells). For values of $k_{1} a_{\text {esr }}$ below 0.5 , there was no averaging. In this region, there is no interference structure. Furthermore, since the wavelength is generally much greater than any feature on the object, the scattering is much less sensitive to irregularities and orientation. In fact, for the ideal spherical shell, the scattering in this region depends upon the volume of the shell (Goodman and Stern, 1962). The scattering by fluid objects (elastic bodies are fluidlike in this region) of any shape in this region also only depends upon the volume (not shape) of the body. In order to prevent any biases associated with the averaging, averaging was not performed in this lower $k_{1} a_{\text {esr }}$ region.

Certainly, this approach of averaging over size and shell thickness is not rigorous and it is applied strictly to obtain the observed effect of smoothed out structure due to the averaging of data over orientation. However, there is some formal basis for the averaging over size and shell thickness. As shown in Stanton et al. (1998b) and summarized in Eqs. (6)(9), the scattering has explicit components that depend upon the local radius of curvature of the curved surface facing the transceiver $\left(f_{\text {spec }}\right.$ and $\left.f_{\text {op }}\right)$. This radius depends (implicitly) upon orientation. Thus an average over a range of radii of curvature of the irregular object is, indeed, connected to the process of averaging over angle of orientation. A similar argument can be made for terms in Eqs. (6)-(9) that depend upon shell thickness which, in turn, depends upon orientation. For example, in a more rigorous treatment, $\mathcal{R}_{12}$ is replaced by a term that depends upon shell thickness (Marston, 1992). There are, however, many complexities in the scattering process that cannot be taken into account with this approach, such as orientation-dependent effects associated with the discontinuity of the shell. What is especially important in use of this averaging approach is recognizing that the return associated with the specular reflection from the front interface $\left(f_{\text {spec }}\right)$ is relatively strong compared with the other ones. If that were the only wave returning from the target, then the averaging process over size and shell thickness would strongly resemble the average over orientation. Although there may be larger errors associated with the other terms, the variation over size in the averages varies the phases of the different terms and creates the desired effects of smoothing out the structure.

In addition to the data to be presented in this section, there is strong empirical evidence in other independent studies that the averaging over size and shell thickness is a reasonable approximation. For example, in a study by Thorne et al. (1995), an average over sizes of the exact modal-series solution for solid elastic spheres was compared with data involving irregular solid elastic objects over a wide range of $k_{1} a_{\text {esr }}$ (Fig. 9 of that paper). The data from the objects involved an average over angle of orientation and sometimes over a distribution of size. There was excellent agreement between the approximate predictions and data. Their analysis demonstrated that, although the approach is not formally correct, it is a reasonable approach toward describing the average scattering for this very complex class of problems.

In this current analysis there was reasonable agreement

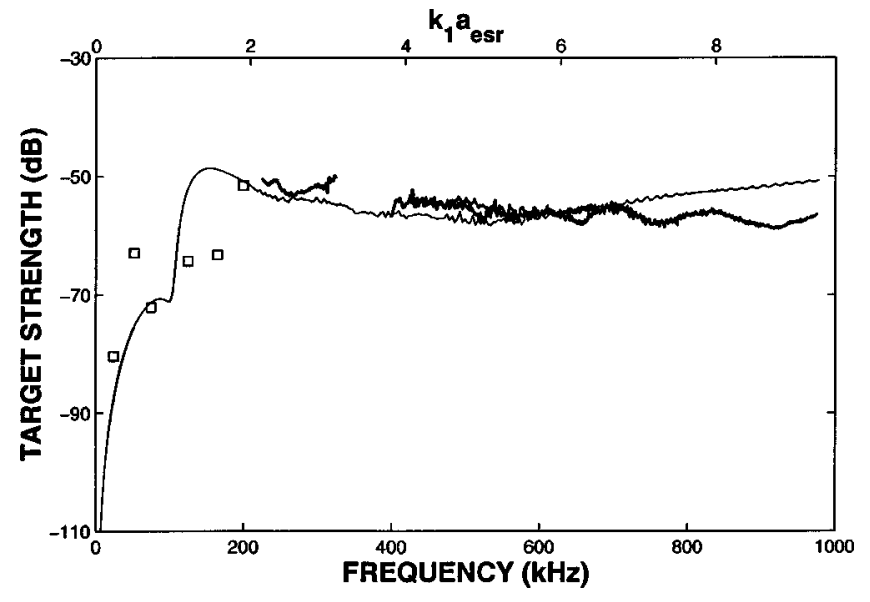

FIG. 11. Comparison between modal-series solution for fluid-filled spherical shell averaged over range of sizes and shell thicknesses with the measured backscattering by an individual periwinkle averaged over all angles of orientation. The average over size and shell thickness (done for $k_{1} a_{\mathrm{esr}}$ $>0.5$ ) is intended to partially emulate the range of radii of curvature and shell thicknesses experienced by the various scattered rays over all angles of orientation. Since the tissue of the body was removed from the shell, the interior fluid of the shell is modeled in this case to be the same as the exterior fluid. Same data as presented in Fig. 5 (animal 97-1, 6.2-mm long). Since the data did not vary significantly with orientation at $24 \mathrm{kHz}$, only the single datum collected at $0^{\circ}$ orientation is plotted at that frequency. Spherical shell solution taken directly from Goodman and Stern (1962). Parameters for the predictions are $\bar{a}=a_{\mathrm{esr}}=2.28 \mathrm{~mm}$ (that is, the average radius used in the predictions was set equal to the measured equivalent spherical radius of the animals; the $a_{\text {esr }}$ shown in the plot are the two terms $\bar{a}=a_{\mathrm{esr}}$ ), $g=2.65, h c=4.35$ (compressional sound speed contrast in shell), $h s$ $=2.24$ (shear sound speed contrast in shell), and shell thickness $=0.2 \mathrm{~mm}$ (from average measured thickness) (material properties from Carmichael, 1982). Here the fractional shell thickness $=($ shell thickness) $/ \bar{a}=0.09$ (where $\bar{a}$ is the average radius used in the predictions). Both the radius and shell thickness were varied in the averages (Gaussian distributed) with a 10\% standard deviation (s.d.) and spanning values within \pm 2 s.d. of the means.

between the averaged modal-series-based solution and the data averaged over all angles of orientation (Fig. 11). The model parameters were based either on direct measurements or published values (no parameters were adjusted to improve the fit to the data). There was reasonable agreement over much of the range of $k_{1} a_{\mathrm{esr}}$ (the Rayleigh and geometric region) including the position of the Rayleigh/geometric transition region. However, there were also some significant deviations. There was a strong resonance in the data near $k_{1} a_{\text {esr }}=0.5$ that could not be predicted with this scattering approach (Figs. 11 and 12). There were also some disagreements near the Rayleigh/geometric transition region and at the highest values of the $k_{1} a_{\mathrm{esr}}$. The latter two regions were very sensitive to the choice of scattering parameters. By varying average shell thickness and bulk material properties (neither were known accurately) in the simulations, different parts of the data could be predicted, but never all simultaneously (not shown). Hence the parameters used for Fig. 11 were published values of material properties and average measured shell thickness.

\section{Modeling the low $k_{1} a_{\text {esr }}$ resonance}

As discussed above, there were strong scattering levels observed near $k_{1} a_{\mathrm{esr}}=0.5$. The levels were observed with 


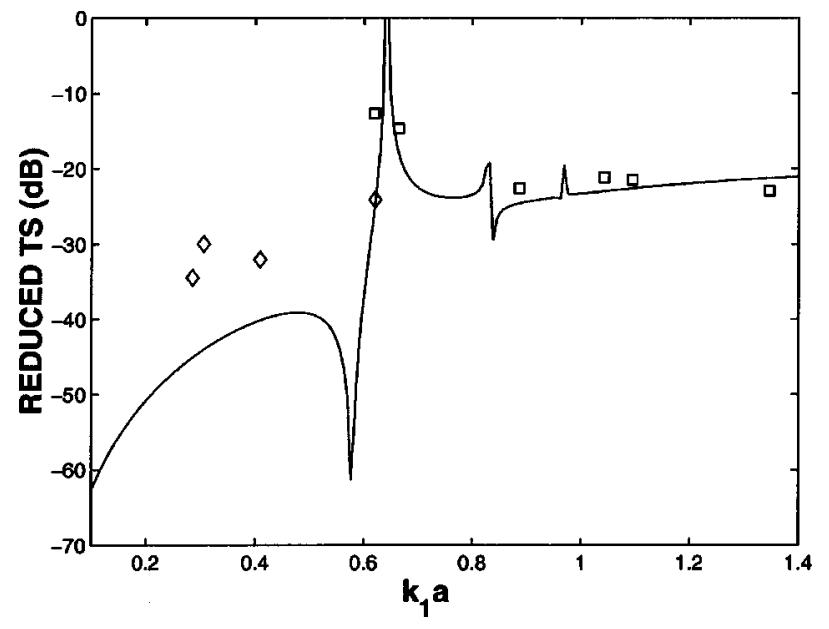

FIG. 12. Modeling study of the resonance structure measured near $k_{1} a_{\text {esr }}$ $=0.5$ (which is at about $k_{1} a=0.6$ on this plot). Six individual periwinkles were used (Nos. 97-1 through 97-6) and two frequencies [24 kHz (diamond) and $50 \mathrm{kHz}$ (square)]. In order to achieve a fit between the model predictions and the data in this exploratory study, the shell thickness in the predictions needed to be approximately $1 / 5$ that of the average measured shell thickness, while the modeling radius " $a$ " needed to be slightly larger than the equivalent spherical radius of the animal. A single realization of the modal-series solution described in Fig. 11 was used with $g=2.65, h c$ $=4.35$, $h s=2.24$, and fractional shell thickness $=($ shell thickness $) / a$ $=0.014$. The values of $a_{\text {esr }}$ given in Table I for the animals were multiplied by 1.25 for use in $k_{1} a$ and $R T S$ in the plot of data. All data correspond to $0^{\circ}$ orientation (i.e., the apex was aimed at the transducers).

repeated measurements of the same animal for replication as well as with a different sized animal. The levels could not be predicted using the modal-series-based approach used above with the average shell thickness values.

We explored the low $k_{1} a_{\text {esr }}$ resonance with the modalseries approach by varying parameters outside of the measured range and found that, for a very thin shell and use of a radius that is slightly larger than the equivalent spherical radius, one could predict a resonance in that region (Fig. 12). In that study, the thickness of the simulated shell was 0.014 times the simulated radius of the shell. This corresponds to a shell that has a thickness that is roughly 1/5 that of the average thickness of the actual measured shell. This observation could lead to two possibilities: (1) Since the shell has a variable thickness, perhaps there are sections that are thin enough to produce such a resonance, (2) since the shell is not spherical and hollow, but rather has internal structure, the structure provides a mechanism for altering the resonance characteristics of the shell.

\section{APPLICATION TO ACOUSTIC SCATTERING IN THE OCEAN}

In spite of the fact that the scattering by the shelled benthic and planktonic animals is strong, there have been few quantitative studies of the acoustic scattering by such animals in the ocean. Below are brief summaries and discussions of applications presented in other papers of simplified versions of these acoustic scattering models to sound scattering by the seafloor and water volume when shelled animals are present.

\section{A. Seafloor}

The scattering by a seafloor covered with shelled animals has been predicted using the results from the periwinkle experiment described herein and compared with data collected in the ocean. The analysis is described in Stanton (2000) and will only be briefly summarized and discussed in the context of the results presented in this paper. In that paper, the key assumption was that first-order scattering from the top layer of shells dominated the scattering. Higher-order scattering, shadowing, and contributions from the seafloor were explicitly ignored which limited the analysis (although note that the Lambert's Law formula implicitly takes these effects into account to some extent). In spite of these limitations, the formulation provided insight into the scattering problem. With these assumptions, the area scattering strength $S_{A}$ of the seafloor at high frequencies was expressed quite simply, in Lambert's Law form, as

$$
S_{A}=\left\langle\operatorname{RTS}^{(m)}\right\rangle+10 \log \mathcal{F}+10 \log \sin ^{2} \theta_{g},
$$

where $\left\langle\operatorname{RTS}^{(m)}\right\rangle$ is the reduced target strength based on the average scattering properties (measured or modeled) of an individual animal, $\mathcal{F}$ is the packing factor of the layer of animals (fraction of area of seafloor covered by the animals), and $\theta_{g}$ is the grazing angle $\left(\theta_{g}=90^{\circ}\right.$ corresponds to normal incidence). What is important about this equation is the fact that the area scattering strength is expressed in terms of the reduced target strength of the animals rather than the target strength. As defined before, the reduced target strength is the target strength normalized by size of the animal. For high $k_{1} a_{\mathrm{esr}}$, the reduced target strength (based on the average scattering properties) is not only independent of size, but, on average, relatively independent of frequency. Thus for geometric scattering, the area scattering strength is relatively independent of acoustic frequency and size of feature on the seafloor. Because of this independence, to a first approximation, the data and modeling for one size of animal can possibly be applied to scattering by surfaces containing other sizes (provided that they too are in the geometric scattering region).

Equation (14) and geometric scattering data in this paper (Fig. 11) were used to make predictions for area scattering strength and compared with scattering data collected by Jackson et al. (1986) and Stanic et al. (1989). In those papers, data were presented involving sound scatter at 20-50 and $20-180 \mathrm{kHz}$, respectively, by sections of the seafloor that were covered with a dense layer of shells. Although the size distribution of shells was not documented in the Jackson et al. (1986) paper and the sizes reported in the Stanic et al. (1989) paper were different than those used in this experiment, our values of reduced target strength (based on average scattering properties) were used because of the independence of $S_{A}$, to first order, upon size. The predictions were within the range of values observed by Jackson et al. (1986) of area scattering strength near normal incidence and slightly higher than the normal incidence values extrapolated via Lambert's Law from the Stanic et al. (1989) data (in the geometric scattering region where the frequencies were above $60 \mathrm{kHz}$ ) which involved shallow grazing angles. This reasonable comparison showed that, in spite of the many assumptions 
made in the surface scattering formulation, the reduced target strength data (or equivalently, the reduced target strength predictions) could robustly make the seafloor scattering predictions for a limited range of conditions. These results show promise of applying normalized scattering data or model from one type of (individual) benthic animal to seafloor regions containing aggregations of another type.

\section{B. Volume}

The scattering by planktonic shelled animals has been predicted using an earlier version of the models described here and compared with acoustic survey data. The analysis is presented in detail in Wiebe et al. (1996) and will only be briefly summarized below in the context of the results presented in this paper. In that paper, a survey using a $420-\mathrm{kHz}$ echosounder over the Georges Bank (near Cape Cod, MA) is described. Gastropods with numerical densities of up to 1000 's $\mathrm{m}^{-3}$ were observed and sometimes dominated the echoes (volume scattering strength of up to $-56 \mathrm{~dB}$ ). In the analysis, a "high-pass" model from Stanton et al. (1994) was used which incorporated the low $k_{1} a$ limit based on a fluid sphere modal-series solution for low frequencies and a ray solution [specular component given in Eq. (7) of this paper] for the high frequencies. Since all data were in the geometric scattering region, only the high $k_{1} a_{\text {esr }}$ portion was taken advantage of in the research. Acoustic scattering predictions were made based upon size and taxa of animals caught in net tows. The predictions were compared with data collected in the $(420-\mathrm{kHz})$ acoustic surveys from the same body of water. There was very good agreement between predictions and observed volume scattering strengths. The slope of the plot of predicted values versus observed data was essentially identical to the idealized expected slope of unity, although there was roughly a $3.5-\mathrm{dB}$ offset between predictions and observations. In some of the regions, it is estimated that the $(2 \mathrm{~mm})$ gastropods dominated the scattering, which is useful in validation of the modeling approach.

The near agreement between the predictions and observations using the very simple approach in that analysis was promising. The simple model, which has no structure in the high $k_{1} a_{\text {esr }}$ region, apparently was reasonable in estimating the scattering by the aggregation of scatterers. As shown in Fig. 11 of this paper, when the scattering is averaged over a distribution of sizes and/or orientations, the high $k_{1} a_{\text {esr }}$ portion of the scattering tends to become smooth. Thus some of the details that are brought out in the more complex model in Eq. (6) may not be very important in the aggregation average. The exception to that observation is that, although the structure may be washed out in the average over size and orientation, the various waves that give rise to the structure have substantial energy and that energy will contribute to the overall scattering levels.

Finally, since the data presented in Wiebe et al. (1996) were only at one (high $k_{1} a_{\text {esr }}$ ) frequency, the low $k_{1} a_{\text {esr }}$ portion of the simplified model could not be tested. However, the results presented in Fig. 11 of this paper indicate that the averaged scattering near the Rayleigh/geometric transition region has enough structure that the simple model used in Wiebe et al. (1996) most likely will not suffice. A more gen- eral model that incorporates the elastic shelled properties of the body is required, such as the one presented in this paper.

\section{SUMMARY AND CONCLUSIONS}

The backscattering data collected with both the benthic animals described here and the planktonic ones described in previous studies indicate the great complexity of the scattering processes involved. The fact that the scattering is so strongly dependent upon angle of orientation shows that the irregularity of the shell needs to be taken into account. Both the spectral and temporal (compressed pulse) analyses provided valuable information regarding the dominant scattering processes. In the high $k_{1} a_{\text {esr }}$ region, there were typically two or more rays scattered back toward the receiver. One of the rays was the specular return off of the front interface while the other was either a ray from within the opercular opening, a subsonic Lamb wave, or other rays not quantified in the analysis. The Rayleigh and Rayleigh/geometric scattering regions were also complicated.

There was reasonable success in predicting the scattering by use of both ray-based and modal-series-based models. Depending upon orientation, the ray-based models used either the echo from the front interface and a Lamb wave or the echo from the front interface and a ray from the opercular opening. Other orientations involved scattering patterns that were more complex than a simple two-ray model could predict. The modal-series solution was averaged over a range of sizes and shell thicknesses to produce reasonable predictions of the data when averaged over angle of orientation. The average of the sizes and thicknesses seemed to at least partially account for the range of curvature and thicknesses seen by the incident acoustic waves over the range of angles. What was especially remarkable in the (periwinkle) analysis was that the ray model involving the Lamb wave used all but three of the parameters used in the analysis involving the smaller gastropods. The three parameters were animal size (which naturally changes with animal), $F_{\text {spec }}$ (which was expected to change with orientation angle), and Lamb wave speed (which was expected to change with the higher $k_{1} a_{\text {esr }}$ and relative shell thickness). Thus the parameters appropriate for the planktonic gastropods which have a similar shape, but disproportionately thinner shell, appear to be applicable to the benthic gastropods.

Application of the data and modeling involving the periwinkles to the problem of scattering by the seafloor were discussed. The results from another paper were summarized which used the measured reduced target strength of the periwinkles to predict scattering by shell-covered sections of the seafloor. The predictions were close to or comparable to the observations, which is a remarkable result considering the differences (or possible differences) between the periwinkles and the animals in the seafloor experiments. Given the fact that the area scattering strength at high frequencies is, to first order, independent of size of (volumetric) feature when a covering exists, this study showed the promise in using reduced target strength data or models such as those presented in this paper to help predict sound scattering by shellcovered seafloors. 
Applications of the scattering models to planktonic shelled animals were also discussed. The results of an earlier paper were summarized, in which a greatly simplified version of the models presented in this work produced reasonable predictions of the scattering observed in an acoustic survey of a region in which planktonic gastropods dominated the scattering. However, that work involved only a single high frequency (geometric scattering) and scattering by ensembles of animals. Work involving lower frequencies and/or echoes involving single animals would require use of the more sophisticated models.

In conclusion, through analysis and modeling of the extensive data set presented herein, great progress has been made toward the understanding of the complex nature of the scattering by benthic and planktonic shelled animals. A better understanding of the dominant scattering mechanisms has been achieved for these complex bodies as well as scattering models developed or extended with conditions of validity determined.

\section{ACKNOWLEDGMENTS}

The authors are grateful to Erhan Mutlu of the Institute of Marine Sciences, Middle East Technical University, Erdemli, Icel, Turkey, for assisting in the measurement of the periwinkle shells during his visit to the Woods Hole Oceanographic Institution (WHOI) in 1997 and Shirley Barkley and Sheila Hurst of WHOI, Woods Hole, MA, for preparing the manuscript to this article. This work was supported by the U.S. Office of Naval Research Grant Nos. N00014-95-10287 and N00014-96-1-0878, and the MIT/WHOI Joint Graduate Education Program. This is contribution number 9794 for the Woods Hole Oceanographic Institution.

Bowman, J. J., Senior, T. B. A., and Uslenghi, P. L. E., Eds. (1987). Electromagnetic and Acoustic Scattering by Simple Shapes (Hemisphere, New York).

Carmichael, R. S., Ed. (1982). CRC Handbook of Physical Properties of Rocks (CRC, Boca Raton, FL), Vol. II.

Chu, D., and Stanton, T. K. (1998). "Application of pulse-compression techniques to broadband acoustic scattering by live individual zooplankton,', J. Acoust. Soc. Am. 104, 39-55.

Chu, D., Stanton, T. K., and Wiebe, P. H. (1992). "Frequency dependence of sound backscattering from live individual zooplankton,' ICES J. Mar. Sci. 49, 97-106.

Felsen, L. B., and Lu, I. T. (1989). "Ray treatment of wave propagation on thin-walled curved elastic plates with truncations,' J. Acoust. Soc. Am. 86, 360-374.

Foote, K. G. (1980). "Importance of the swimbladder in acoustic scattering by fish: A comparison of gadoid and mackerel target strengths," J. Acoust. Soc. Am. 67(6), 2084-2089.

Goodman, R. R., and Stern, R. (1962). "Reflection and transmission of sound by elastic spherical shells,' J. Acoust. Soc. Am. 34, 338-344. For modal-series coefficients involving spherical shells with an interior fluid different than that of the exterior fluid see, for example, Stanton, T. K. (1990). "Sound scattering by spherical and elongated, shelled bodies," ibid. 88, 1619-1633.

Ho, J. M. (1994). "Geometrical theory of acoustic scattering by thin elastic shells,', J. Acoust. Soc. Am. 96, 3115-3125.

Ho, J. M., and Felsen, L. B. (1990). "Nonconventional traveling wave formulations and ray-acoustic reductions for source-excited fluid-loaded thin elastic spherical shells,', J. Acoust. Soc. Am. 88, 2389-2414.

Jackson, D. R., Baird, A. M., Crisp, J. J., and Thomson, P. A. G. (1986). "High-frequency bottom backscatter measurements in shallow water,' J. Acoust. Soc. Am. 80(4), 1188-1199.
Jebsen, G. M., and Medwin, H. (1982). "On the failure of the Kirchhoff assumption in backscatter,' J. Acoust. Soc. Am. 72, 1607-1611.

Kaduchak, G., Kwiatkowski, C. S., and Marston, P. L. (1995). "Measurements and interpretation of the impulse response for backscattering by a thin spherical shell using a broad-bandwidth source that is nearly acoustically transparent,' J. Acoust. Soc. Am. 97, 2699-2708.

Kargl, S. G., and Marston, P. L. (1989). "Observations and modeling of the backscattering of short tone bursts from a spherical shell: Lamb wave echoes, glory, and axial reverberations,' J. Acoust. Soc. Am. 85, 10141028

Lalli, C. M., and Gilmer, R. W. (1989). Pelagic Snails (Stanford University Press, Stanford), Chap. 4.

Lowenstam, H. A. (1954). "Factors affecting the aragonite: calcite ratios in carbonate-secreting marine organisms,"' J. Geol. 62, 284-322.

Marston, P. L. (1992). "Geometrical and catastrophe optics methods in scattering," in Physical Acoustics, edited by A. D. Pierce and R. N. Thurston (Academic, New York), Vol. 21.

Norris, A. N., and Rebinsky, D. A. (1994). "Acoustic coupling to membrane waves on elastic shells,", J. Acoust. Soc. Am. 95, 1809-1829.

Rebinsky, D. A., and Norris, A. N. (1995). "Benchmarking an acoustic coupling theory for elastic shells of arbitrary shape,' J. Acoust. Soc. Am. 98, 2368-2371.

Stanic, S., Briggs, K. B., Fleischer, P., Sawyer, W. B., and Ray, R. I. (1989). "High frequency acoustic backscattering from a coarse shell ocean bottom,', J. Acoust. Soc. Am. 85(1), 125-136.

Stanton, T. K. (1990). "Sound scattering by spherical and elongated shelled bodies,' J. Acoust. Soc. Am. 88, 1619-1633.

Stanton, K. T. (2000). "On acoustic scattering by a shell-covered seafloor," J. Acoust. Soc. Am. 108, 551-555.

Stanton, T. K., Chu, D., and Wiebe, P. H. (1996). "Acoustic scattering characteristics of several zooplankton groups," ICES J. Mar. Sci. 53, 289-295.

Stanton, T. K., Chu, D., Wiebe, P. H., Martin, L. V., and Eastwood, R. L. (1998a). "Sound scattering by several zooplankton groups. I. Experimental determination of dominant scattering mechanisms," J. Acoust. Soc. Am. 103, 225-235.

Stanton, T. K., Chu, D., and Wiebe, P. H. (1998b). "Sound scattering by several zooplankton groups. II. scattering models,' J. Acoust. Soc. Am. 103, 236-253.

Stanton, T. K., Wiebe, P. H., Chu, D., Benfield, M., Scanlon, L., Martin, L., and Eastwood, R. L. (1994). "On acoustic estimates of zooplankton biomass,' ICES J. Mar. Sci. 51, 505-512.

Thorne, P. D., Hardcastle, P. J., and Soulsby, R. L. (1993). "Analysis of acoustic measurements of suspended sediments,', J. Geophys. Res. 98(C1), 899-910.

Thorne, P. D., Waters, K. R., and Brudner, T. J. (1995). "Acoustic measurements of scattering by objects of irregular shape," J. Acoust. Soc. Am. 97(1), 242-251.

Überall, H. (1973). "Surface waves in acoustics,', in Physical Acoustics, edited by W. B. Mason and R. N. Thurston (Academic, New York), Vol. 10 , pp. $1-60$

Wiebe, P. H., Mountain, D. G., Stanton, T. K., Greene, C. H., Lough, G., Kaartvedt, S., Dawson, J., and Copley, N. (1996). "Acoustical study of the spatial distribution of plankton on Georges Bank and the relationship between volume backscattering strength and the taxonomic composition of the plankton,', Deep-Sea Res. II 43(7-8), 1971-2001.

Wiebe, P. H., Stanton, T. K., Benfield, M. C., Mountain, D. G., and Greene, C. H. (1997). "High-frequency acoustic volume backscattering in the Georges Bank coastal region and its interpretation using scattering models,' IEEE J. Ocean Eng. 22(3), 445-464.

Yang, Y., Norris, A. N., and Couchman, L. S. (1995). "Acoustic scattering from fluid-loaded elastic shells: A Gaussian beam approach,' J. Acoust. Soc. Am. 98, 611-622.

Yang, Y., Norris, A. N., and Couchman, L. S. (1996). "Ray tracing over smooth elastic shells of arbitrary shape,' J. Acoust. Soc. Am. 99, 55-65.

Zhang, L. (1996). "High-Frequency Seafloor Acoustic Backscattering with the Presence of Turbulence in Seawater,' Ph.D. dissertation, University of Southern Mississippi. 